\title{
Topological Defects in the Abrikosov Lattice of Vortices in Type-II Superconductors
}

\author{
M.J.W. Dodgson and M.A. Moore \\ Theoretical Physics Group \\ Department of Physics and Astronomy \\ The University of Manchester, M13 9PL, UK
}

(29 ${ }^{\text {th }}$ November 1994)

\begin{abstract}
The free energy costs for various defects within an Abrikosov lattice of vortices are calculated using the lowest Landau level approximation (LLL). Defect solutions with boundary conditions for lines to meet at a point (crossing defect) and for lines to twist around each other (braid defect) are sought for 2, 3, 6, and 12 lines. Many results have been unexpected, including the nonexistence of a stable two- or three-line braid. This, and the high energy cost found for a six-line braid lead us to propose that the equilibrium vortex state is not entangled below the irreversibility line of the high- $T_{c}$ superconductors or in a large part of the vortex-liquid phase above this line. Also, the solution for an infinite straight screw dislocation is found, and used to give a limiting form for the free energy cost of very large braids. This depends on the area enclosed by the braid as well as its perimeter length.
\end{abstract}

PACS no.s: 74.60.Ge; 74.20.De

Typeset using REVTEX 


\section{INTRODUCTION}

There has been a lot of interest in the effects of thermal fluctuations on the behaviour of flux-lines ever since the discovery of the high- $T_{c}$ superconductors. The high temperatures and fields at which these new materials remain in the mixed state, and their small coherence lengths, mean that these fluctuations may be strong enough to totally alter the nature of the vortex state from the mean-field solution of an Abrikosov lattice. Debate and controversy has continued on the possibilities of different vortex phases above and below the irreversibility line (at which the vortices become depinned). We believe that the results of the calculations in this paper shed some light on certain questions on the vortex state. Our primary conclusion is that there is very little entanglement near and below the irreversibility line.

In this work, we start with the triangular vortex-lattice, and consider the free energy costs of topological defects within the lattice. Because of thermal fluctuations, the spontaneous formation of defects of finite free energy cost is important in the description of the vortex state. These defects may include 'crossings' where two or more lines may pass through the same point, and 'braids' where a collection of lines twist around each other as one moves along the field direction. Our approach is always to begin with the Abrikosov lattice of vortices that corresponds to the mean-field solution for the Ginzburg-Landau free energy functional within the lowest Landau level approximation 1 .2. We realize of course that the LLL approximation only holds strictly in the high field regime of the superconducting phase diagram, just below $H_{c 2}$, and even that the existence of this lattice may be questioned. Having set up the perfect lattice of vortices, we than allow a restricted number of the vortex-lines to move subject to certain boundary conditions that define a topological defect, and then find the minimum free energy cost of such a defect.

The free energy cost of a crossing configuration provides an estimate of the energy barrier for vortex lines to cross through each other, or to cut and reconnect. Accurate estimates of the crossing energy barrier in the different regions of the $H-T$ phase diagram are desired because of their relevance to the vortex dynamics of high- $T_{c}$ superconductors. The energy barrier. $U_{x}$, to crossing/cutting of vortex lines is a fundamental parameter of the entangled state 3 . This has encouraged calculations of this energy in different approximation 5.6. The calculation in this paper is the first to consider the effects on $U_{\times}$of the surrounding triangular lattice.

The braid defects are examples of the sort of configurations one expects to find in the entangled vortex state. As well as affecting the response to pinning centers, they will have interactions with any component of current parallel to the applied field. The growth of braid defects may be a source of dissipation of such longitudinal currents. An important result of our calculation is that there are no stable two-line or three-line braid defects, at least within our approximations. The smallest braid defect that is stable, but which has a relatively high free energy cost, is the six-line braid. The non-existence of stable braid configurations with small free energies has the consequence that in a sample of typical size of high- $T_{c}$ superconductor there is a large region of the $H$ - $T$ phase diagram at which there will be no braid defects present in thermal equilibrium. We estimate that there will be no braids for values of the reduced temperature far above the irreversibility line and quite close to the $H_{c 2}$ line (see Section $\mathrm{VA}$ ). If there are no braid defects present in a given sample then 
the vortex system is disentangled. This is contrary to the picture of an entangled vortex liquid suggested by Nelson $\mathrm{B}$.

In Section [1] we describe the formulation of our calculations. Section III] contains our calculations and results for crossing and braid defects involving two, three, six and twelve lines. In Section [D we extend our method to large scale defects of the Abrikosov lattice. The free energy cost of an infinite straight screw dislocation is calculated, and the result is used to find a limiting form for the free energy cost of very large braids. This free energy is found to depend on the area enclosed by the braid as well as the perimeter length. In Section $\square$ it is shown how our results for small braids lead to the conclusion that entanglements are not important in a large region above and below the irreversibility line of high- $T_{c}$ superconductors of typical sizes. Other applications of the calculations are also suggested in Section $\square$.

\section{METHOD OF THE CALCULATIONS}

For the sake of clarity, and to set the notation used, the general method behind the calculations is now described. First, the Ginzburg-Landau theory is formulated and the Lowest Landau Level is defined. Then the ground state corresponding to an Abrikosov triangular lattice is presented, and it is shown how to construct deviations from the perfect lattice which remain in the LLL. The thermodynamics of flux-lines within a type-II superconductor are well described for fields $H$ near $H_{c 2}(T)$ by Ginzburg-Landau theory. Within this theory, the free energy density is expanded in terms of the superconducting order parameter and its derivatives,

$$
\mathcal{F}\{\psi\}=\sum_{\mu=1}^{3} \frac{1}{2 m_{\mu}}\left|\left(-i \hbar \partial_{\mu}-2 e \mathrm{~A}_{\mu}\right) \psi\right|^{2}+\alpha|\psi|^{2}+\frac{\beta}{2}|\psi|^{4}+\frac{b^{2}}{2 \mu_{0}}+\text { const },
$$

where $\mathbf{b}=\boldsymbol{\nabla} \times \mathbf{A}$ is the microscopic flux density. This equation applies for general anisotropy but only applies to a homogenous system (layering effects are ignored). For relevance to layered superconductors, we consider a limited anisotropy with $m_{x}=m_{y}=m_{a b}$ and $m_{z}=$ $m_{c}$. For a uniform external field $\mathbf{H}_{0}$ parallel to the z-axis, it is the Gibbs free energy, $\mathcal{G}\{\psi\}=F_{0}+\mathcal{F}\{\psi\}-\mathbf{b} . \mathbf{H}_{0}$ that controls the properties of the system $\mathbf{\theta}$.

Our main approximation (valid for high fields) is to restrict the order parameter to the 'Lowest Landau Level' (LLL) subspace, defined by

$$
\Pi \psi=0,
$$

where $\Pi=\Pi_{x}+i \Pi_{y}, \Pi_{x}=-i \hbar(\partial / \partial x)-2 e A_{x}$, and $\Pi_{y}=-i \hbar(\partial / \partial y)-2 e A_{y}$. Within this restriction, substitution into the Gibbs free energy leads to an effective free energy density of

$$
\mathcal{F}\{\psi\}=\alpha_{H}|\psi|^{2}+\frac{\beta_{K}}{2}|\psi|^{4}+\frac{\hbar^{2}}{2 m_{c}}\left|\frac{\partial \psi}{\partial z}\right|^{2},
$$

where $\alpha_{H}=\alpha+\left(e \hbar / m_{a b}\right) \mu_{0} H_{0}$, and $\beta_{K}=\beta-\mu_{0}\left(e \hbar / m_{a b}\right)^{2}$. Here, $\alpha_{H}$ is our reduced temperature variable, which is negative below the $H_{c 2}$ line and positive above. It is useful to make the transformations 


$$
\begin{aligned}
& \psi \rightarrow \tilde{\psi}=\left(\frac{\beta_{K}}{\left|\alpha_{H}\right|}\right)^{\frac{1}{2}} \psi \\
& z \rightarrow h=\left(\frac{2 m_{c}\left|\alpha_{H}\right|}{\hbar^{2}}\right)^{\frac{1}{2}} z .
\end{aligned}
$$

Thus, $h$ is the distance along the $c$-axis in units of the mean field correlation length in this direction, $\xi_{c}=\sqrt{\hbar^{2} / 2 m_{c}\left|\alpha_{H}\right|}$. Substituting (2.4) into (2.3) and integrating over all space (for an infinite bulk superconductor) gives a total free energy

$$
F=\left(\frac{\hbar^{2}}{2 m_{c}}\right)^{\frac{1}{2}} \frac{\left|\alpha_{H}\right|^{\frac{3}{2}}}{\beta_{K}} \int d h \int d^{2} r\left\{-|\tilde{\psi}|^{2}+\frac{1}{2}|\tilde{\psi}|^{4}+\left|\frac{\partial \tilde{\psi}}{\partial h}\right|^{2}\right\}
$$

A consequence of using the LLL approximation is that solutions of (2.2) have the general form in the plane perpendicular to the magnetic field

$$
\psi_{0}(x, y)=f(z) e^{-\pi y^{2} / \eta}
$$

where $z$ is not the third dimension in space, but the complex variable, $z=x+i y$, and $\eta=\Phi_{0} / B,\left(B \equiv\langle b\rangle\right.$ is the mean magnetic flux density, and $\Phi_{0}=h / 2 e$ is the flux quantum $)$. $f(z)$ is an analytic function of $z$. Any function of the above form is a function within the LLL subspace. $f(z)$ may be written quite generally in a product form

$$
f(z) \propto \prod_{i}\left(z-z_{i}\right)
$$

where the zeros in $f(z), z=z_{i}$, correspond to the positions of vortices within the superconductor. Of course it is well known that the order parameter that minimizes the free energy (2.3) in the mixed state is a triangular periodic lattice of flux-lines2. The corresponding function $f(z)$ for such a lattice is a Jacobi theta function 1

$$
\psi_{0}(x, y)=\phi(\mathbf{r} \mid \mathbf{0})=C e^{-\frac{\pi y^{2}}{\eta}} \vartheta_{3}\left(\frac{\pi z}{l}, \frac{\pi \tau}{l}\right) .
$$

Properties of $\phi(\mathbf{r} \mid \mathbf{0})$ and related functions $\phi\left(\mathbf{r} \mid \mathbf{r}_{0}\right)$ that span the LLL subspace are given in $\mathbf{1}$. The function $|\phi(\mathbf{r} \mid \mathbf{0})|^{2}$ has the periodicity $\mathbf{r}_{I}$ and $\mathbf{r}_{I I}$ where $\mathbf{r}_{I}=l(1,0), \mathbf{r}_{I I}=l(1 / 2, \sqrt{3} / 2)$, and $\tau=x_{I I}+i y_{I I}=l / 2+i l \sqrt{3} / 2 . l$ is the distance between neighboring zeros (vortices) in $\phi\left(\mathbf{r} \mid \mathbf{r}_{0}\right)$. The area of the unit cell must contain one quantum of flux, so that $\eta=l^{2} \sqrt{3} / 2$. The prefactor in (2.8) is found to be $C=3^{1 / 8}$, if we use the normalization condition

$$
\left\langle\left|\psi_{0}\right|^{2}\right\rangle=\int_{\text {unit cell }} \frac{d^{2} r}{\eta}|\phi(\mathbf{r} \mid \mathbf{0})|^{2}=1
$$

To find the free energy of the Abrikosov lattice, we substitute $\tilde{\psi}(x, y, h)=K \psi_{0}(x, y)$ into (2.5). As $\left|\psi_{0}\right|^{2}$ is periodic, we can integrate over one unit cell to find the average free energy density

$$
f=\left(\frac{\hbar^{2}}{2 m_{c}}\right)^{\frac{1}{2}} \frac{\left|\alpha_{H}\right|^{\frac{3}{2}}}{\beta_{K}} \int_{\text {unit cell }} \frac{d^{2} r}{\eta}\left\{-K^{2}\left|\psi_{0}\right|^{2}+K^{4}\left|\psi_{0}\right|^{4}\right\} .
$$


This is minimized with respect to $K$ (using (2.9)) by the condition $K^{2}=1 / \beta_{A}$, where $\beta_{A}$ is the Abrikosov parameter $\beta_{A}=\left\langle\left|\psi_{0}\right|^{4}\right\rangle /\left\langle\left|\psi_{0}\right|^{2}\right\rangle^{2} \simeq 1 \cdot 1596$. The free energy per flux-line per unit length for the Abrikosov lattice is therefore given by $f_{L}=-\alpha_{H}{ }^{2} / 2 \beta_{K} \beta_{A}$.

As any LLL function has the general form (2.7), including $f(z)=\vartheta_{3}(\pi z / l, \pi \tau / l)$ (see Appendix A), we can therefore construct a function representing the order parameter for a triangular lattice with one flux line displaced from $z=z_{0}$ to $z=\zeta_{0}$, by

$$
\psi_{\text {displaced }}(\mathbf{r})=\psi_{0}(\mathbf{r}) \frac{\left(z-\zeta_{0}\right)}{\left(z-z_{0}\right)} .
$$

This cancels out the first order zero in $\psi_{0}$ at $z=z_{0}$ and replaces it with a first order zero at $z=\zeta_{0}$. Because this new function has the form of (2.7), it is still within the LLL subspace, even though it is obviously not the configuration of lowest free energy (which is $\psi_{0}$ ). (A similar method to this was used by Brandt 0 ). (2.11) can be generalized to any number of displaced lines, to form order parameters for various defects of the perfect lattice within the LLL subspace. The free energy cost of any such defect is given by integrating over all space the difference in the free energy densities of the defect and the ground state:

$$
\Delta F_{\text {defect }}=\left(\frac{\hbar^{2}}{2 m_{c}}\right)^{\frac{1}{2}} \frac{\left|\alpha_{H}\right|^{\frac{3}{2}}}{\beta_{A} \beta_{K}} \int d h \int d^{2} r\left\{\mathcal{F}\left(\psi_{\text {defect }}\right)-\mathcal{F}\left(\psi_{l}\right)\right\},
$$

where

$$
\mathcal{F}(\psi)=-|\psi|^{\epsilon}+\frac{\infty}{\epsilon \beta_{\mathcal{A}}}|\psi|^{\triangleright}+\left|\frac{\partial \psi}{\partial\langle}\right|^{\epsilon} .
$$

In all the problems described in this paper, the procedure is to define a defect in the perfect lattice by allowing the positions of some of the vortices to vary with $h$ subject to certain boundary conditions leaving the rest of the vortices fixed in their positions in the triangular lattice. The total free energy of the defect is then minimized with respect to the positions of the chosen vortices, subject to these boundary conditions. This is done by expanding the free energy density as a polynomial in a set of variables that describe the coordinates in the $x-y$ plane of the chosen lines, say $\left\{\zeta_{i}(h)\right\}$ for the vortices labelled by $i$. The coefficients in the expansion will be functions of $x$ and $y$ only (as there is no $h$ dependence of $\psi_{0}$ ). These coefficients must be integrated over the $x-y$ plane to give the free energy per unit length along $h$, that depends on $\left\{\zeta_{i}(h)\right\}$ and their first derivatives. The form of the variables $\left\{\zeta_{i}(h)\right\}$ that minimizes the total free energy will be solutions to Euler-Lagrange non-linear differential equations. By solving these the lowest free energy cost of a given type of defect can be found.

One of our main approximations is that we do not allow the lines not directly involved in the defect to move in response to the presence of the defect (i.e. the relaxation of the surrounding lattice). This may seem like a poor approximation that will seriously over estimate the free energies of the real defects. However, we have found that this is not the case when we allow the nearest neighboring lines to move (as in Section IIIB) and we believe that relaxation of the lattice will only slightly reduce our values of defect free energy costs. The reason for this is that we are considering a lattice of vortex lines, which cost energy to tilt with respect to the field direction, so if we consider a localized defect taking place over 
a small length scale, $L$, then the surrounding lines would have to tilt considerably over the distance $L$ if they are to reduce their interaction energy with the defect lines, and this will cost too much tilt energy.

An important general result of our method is that for any defect of the ground state made from changing the positions of $n$ of the vortices, the free energy cost will diverge logarithmically with the total size of the system, unless the $n$ lines move symmetrically about their "mean midpoint 10 . That is, if $n$ lines move from their ground state values $\left\{z_{i}\right\}$ to the new positions $\left\{\zeta_{i}(h)\right\}$, then the condition for a non-divergent free energy is:

$$
\sum_{i=1}^{n} \zeta_{i}(h)=\sum_{i=1}^{n} z_{i}
$$

i.e. if we define the 'center' of the defect as the vector sum of the coordinates of the ground state lattice position, $z_{i}$, then this condition says that the vectors of the coordinates of the defect line must always sum to this 'center'. An important point to note is the restriction in this derivation to a defect of $n$ lines. If one wanted to consider a defect that by definition could not satisfy the symmetry relation (2.14) then a finite free energy could still be obtained, but only by allowing the 'relaxation' of other lines in the system. The motion of these extra lines would be so as to ensure the symmetry condition for the total number of moving lines, and through this cancel out the logarithmic divergence. However, if it is possible for a simple $n$-line defect to satisfy (2.14), then the free energy cost will depend only on the locally surrounding lattice. We take advantage of this result in the following calculations to reduce the number of free parameters used for describing a given defect.

\section{CALCULATIONS FOR SMALL DEFECTS}

\section{A. Two Lines Crossing}

The first defect to which the above method is applied is the case of two neighboring lines within the Abrikosov lattice moving together to meet at a point. The importance of this defect is that it is the configuration providing an energy barrier to the 'cutting' or 'reconnecting' of two vortices. The simplest way to estimate the energy of two lines crossing is to keep all the surrounding lines in their original positions in the lattice, and to assume that the two lines move symmetrically towards each other (see Fig 1). This avoids a divergence in the free energy cost, and results in an order parameter depending on one parameter only.

The calculation for this simple defect is described in more detail than the other problems in order to demonstrate the general procedure of these calculations. If we take the origin, $O$, of the complex $z$-plane to be at the midpoint of the two lines, then we have two vortices $(=$ zeros in the order parameter) which move from $z= \pm l / 2$ to $z= \pm a(h)$, with the boundary conditions $a( \pm \infty)= \pm l / 2$. Using (2.11), the LLL order parameter for two lines crossing may be written

$$
\psi_{2}(x, y, h)=\psi_{0}(x, y) \frac{(z+a(h))(z-a(h))}{\left(z+\frac{l}{2}\right)\left(z-\frac{l}{2}\right)}
$$


(i)
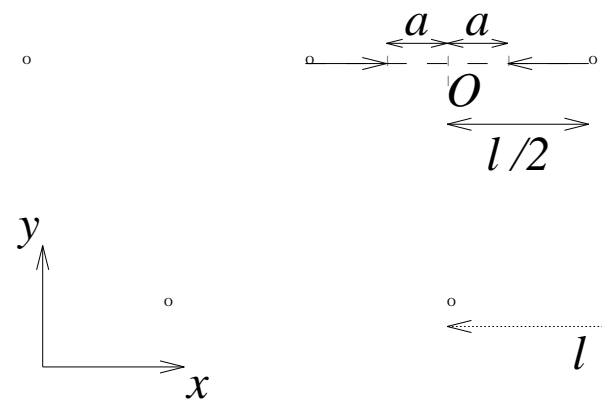

(ii)

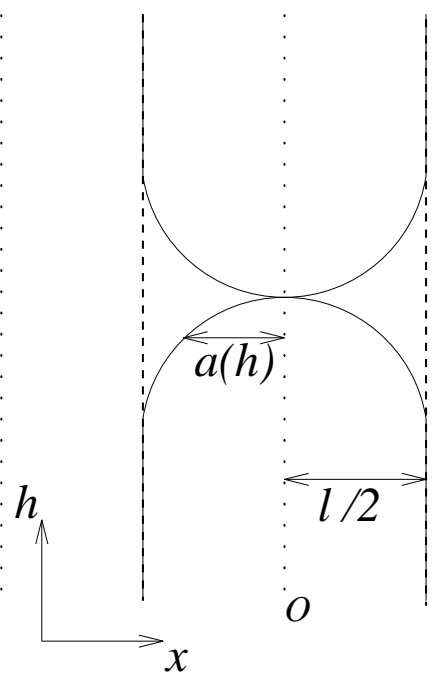

FIG. 1. (i) Cross-section of the vortex lattice on the $x-y$ plane, showing the path along which two neighboring lines may cross. The ground state positions of vortices are marked by the small circles. (ii) Schematic side-view of the vortex lattice with two lines crossing. The solid lines are in the plane of the paper and the dotted lines represent vortices just behind/in front of this plane.

The subscript, 2, indicates the number of lines allowed to deviate from their positions in the ground state triangular lattice. It is now necessary to find an expression for the free energy change of the displaced lines compared to the undistorted lattice as a functional of $a(h)$, then minimize this functional to find the correct shape of the crossing lines (i.e. the configuration of lowest free energy). Looking at (2.12) and (2.13) one sees that we need to expand the differences $\left|\psi_{2}\right|^{2}-\left|\psi_{0}\right|^{2}$, and $\left|\psi_{2}\right|^{4}-\left|\psi_{0}\right|^{4}$, as well as $\left|\partial \psi_{2} / \partial h\right|^{2}$ in terms of $a$. The coefficients of these expressions are then integrated over the $x-y$ plane which leads to a free energy per unit length along the $h$-direction of the form:

$$
\begin{aligned}
f_{2}\{a(h)\} & \equiv \int \frac{d^{2} r}{l^{2}}\left\{\mathcal{F}\left(\psi_{\epsilon}\right)-\mathcal{F}\left(\psi_{1}\right)\right\} \\
& =\sum_{i=0}^{4} c_{i}^{(2)} a^{2 i}+c^{(2)} a^{2}\left(\frac{d a}{d h}\right)^{2},
\end{aligned}
$$

with the coefficients $c_{i}^{(2)}$ equal to the $c_{i 0}^{(2)}$ given in Table 罒, and $c^{(2)} \simeq 94.20 / l^{4}$. For example, the coefficient $c_{1}^{(2)}$ was found by calculating:

$$
\begin{aligned}
c_{1}^{(2)} & =2 \int \frac{d^{2} r}{l^{2}} \frac{\left|\psi_{0}\right|^{2} R e\left(z^{2}\right)}{\left|z^{2}-\left(\frac{l}{2}\right)^{2}\right|^{2}}-\frac{2}{\beta_{A}} \int \frac{d^{2} r}{l^{2}} \frac{\left|\psi_{0}\right|^{4} R e\left(z^{2}\right)|z|^{2}}{\left|z^{2}-\left(\frac{l}{2}\right)^{2}\right|^{4}} \\
& \simeq-5 \cdot 201 / l^{2} .
\end{aligned}
$$

Note that all of the integrals that make up these coefficients must be convergent, as the denominator of the integrands always grows (for large $z$ ) as at least two powers of $|z|$ higher than for the numerator (there is no logarithmically divergent coefficient as the symmetry condition (2.14) is satisfied). The first term in (3.2) represents the potential energy cost per 


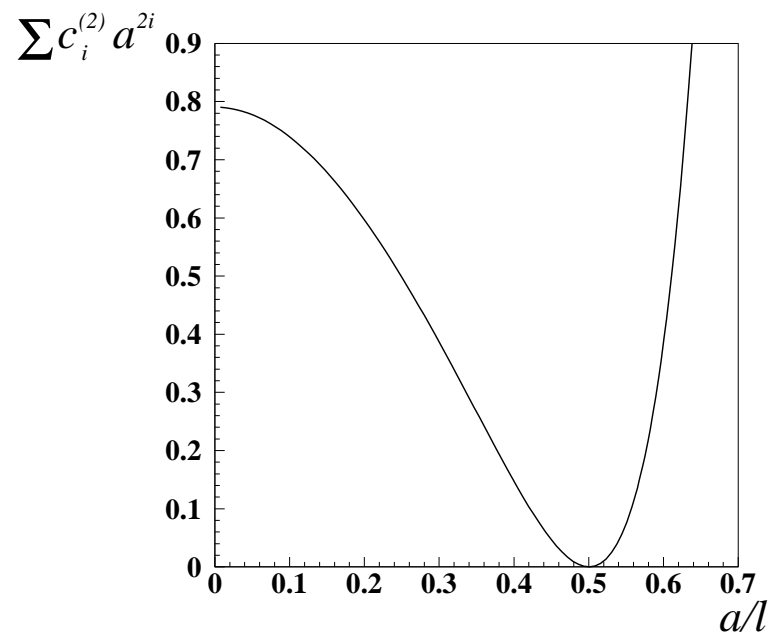

FIG. 2. The potential term, $\sum_{i} c_{i}^{(2)} a^{2 i}$, for two lines crossing.

unit length with the two lines displaced but still straight. The form of this term is shown in Fig 2. The second term is the tilt energy of the two lines. It increases with the distance $a$, which is consistent with an attractive force between the anti-parallel components of the vortex segments in the two lines. One could think of the equation (3.2) in analogy with a Lagrangian density in classical mechanics, of the general form $\mathcal{L}=\mathcal{T}-\mathcal{V}$ for a body moving in one dimension, $a$, and with 'time' equivalent to the $h$-direction. The total free energy is proportional to the integral over all $h$ of (3.2). Applying the Euler-Lagrange equation for stationary values of a functional, and integrating once, one arrives at the equation for the form of $a(h)$ that minimizes the free energy change, $f_{2}-a^{\prime}\left(\partial f_{2} / \partial a^{\prime}\right)=$ const. Substituting (3.2) and applying the boundary conditions gives

$$
c^{(2)} a^{2}\left(\frac{d a}{d h}\right)^{2}=\sum_{i=0}^{4} c_{i}^{(2)} a^{2 i} .
$$

This can now be integrated up from the point of crossing, $a=0$ and (say) $h=0$, to give the correct form of $a(h)$. As $h \rightarrow 0$ this will tend to $a(h) \simeq\left(4 c_{0}^{(2)} / c^{(2)}\right)^{1 / 4} \sqrt{h}$ (this form at

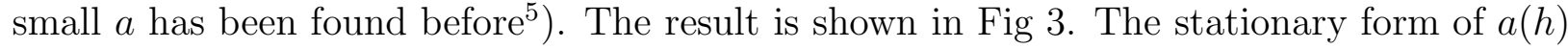
can now be substituted into (3.2) and (2.12), and the integral over $h$ performed to find the total free energy change for crossing.

We find $\int d h f\{a(h)\} \simeq 2 \cdot 32$, which gives

$$
\Delta F_{2 \times}=\left(\frac{\hbar^{2}}{2 m_{c}}\right)^{\frac{1}{2}} \frac{\left|\alpha_{H}\right|^{\frac{3}{2}}}{\beta_{K} \beta_{A}} \frac{2 \Phi_{0}}{\sqrt{3} B} \times 2 \cdot 32 .
$$

To put this in a simpler form, we use the dimensionless factor $\alpha_{T}$ defined by

$$
\alpha_{H}=\left(\frac{\beta_{K} e \mu_{0} H k_{B} T \sqrt{2 m_{c}}}{4 \pi \hbar^{2}}\right)^{\frac{2}{3}} \alpha_{T}
$$




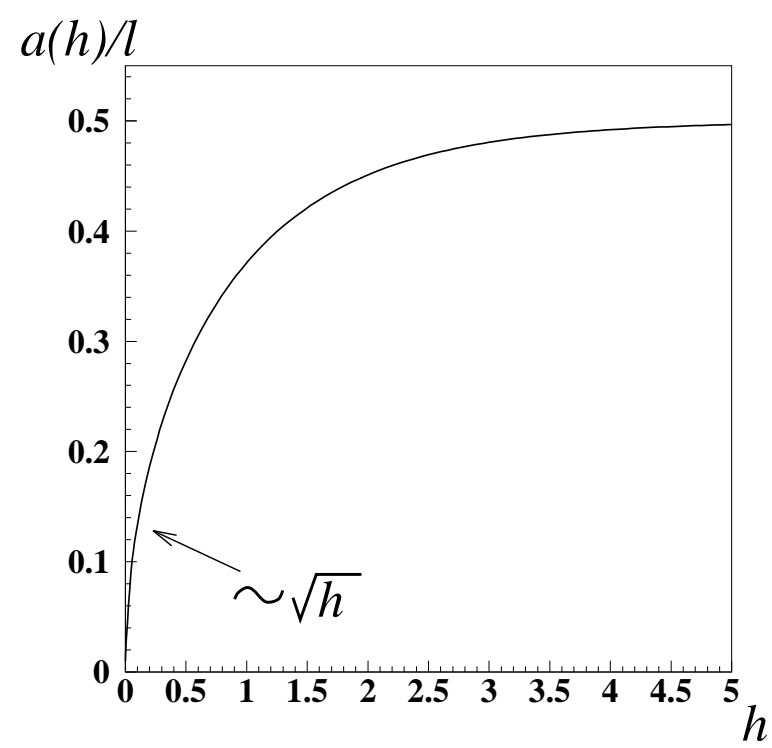

FIG. 3. The form of $a(h)$ that minimizes the free energy subject to the crossing boundary condition that $a(0)=0$. The expected form as $h \rightarrow 0$ is shown.

Substituting (3.7) into (3.6) gives

$$
\Delta F_{2 \times}=0 \cdot 58 k_{B} T\left|\alpha_{T}\right|^{\frac{3}{2}} .
$$

All of the following free energies will also be quoted in this form. However, there is at present a variety of different units used in the literature. For comparison, we can also write $\alpha_{T}$ in terms of the temperature, the field, and the Ginzburg number Gi. (The Ginzburg number is a useful parameter that describes the strength of thermal fluctuations of the superconducting order parameter $\left.{ }^{11}\right)$. We use the definition of $G i$ given by 11 :

$$
G i=\frac{1}{2(8 \pi)^{2}}\left(\frac{2 k_{B} T_{c}}{\mu_{0} H_{c}^{2}(0) \xi_{a b}^{2}(0) \xi_{c}(0)}\right)^{2} .
$$

The factor $(1 / 8 \pi)^{2}$ enters as we change the units of magnetic energy from c.g.s. to S.I. Using standard relations for the coherence length and critical fields we find that:

$$
\left|\alpha_{T}\right|^{\frac{3}{2}}=\frac{\sqrt{2}(1-t+\mathrm{h})^{\frac{3}{2}}}{G i^{1 / 2} \mathrm{~h} t} .
$$

$t$ and $\mathrm{h}$ are the dimensionless temperature and field $t=T / T_{c}$ and $\mathrm{h}=H / H_{c 2}(0)$ where $H_{c 2}(0)$ denotes the straight line extrapolation to zero temperature of the $H_{c 2}$ line when the field is applied along the $c$-axis.

\section{B. Two Lines Crossing with Relaxation of Nearest Lines}

The result in (3.8) is only an upper bound on the actual energy for the two lines to cross. This is because we have ignored any relaxation that the remaining lines in the lattice 


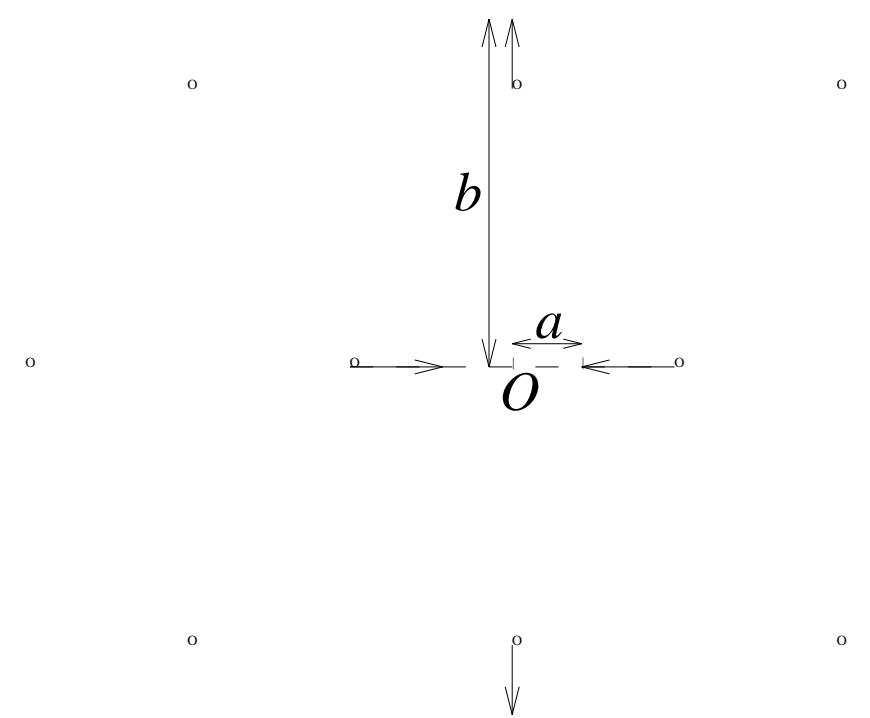

FIG. 4. Cross-sectional view of the lattice when two lines are allowed to cross, and the two nearest neighbors move in response.

may undergo in reaction to the crossing so as to decrease the free energy cost of the defect. In order to test how good an estimate this result is, we now investigate the extent that allowing the surrounding vortices to move may change the free energy. It seems likely that it is the lines closest to the crossing region which will move the most in this defect, and therefore affect the energy of the crossing the most. As a second approximation to (3.8), a calculation was performed where the two lines closest to the crossing center, above and below, were allowed to move symmetrically in response to the crossing (see Fig 4 ). The new order parameter with this allowed motion of the four lines is:

$$
\psi_{4}(x, y, h)=\psi_{0}(x, y) \frac{\left(z^{2}-a(h)^{2}\right)\left(z^{2}+b(h)^{2}\right)}{\left(z^{2}-\left(\frac{l}{2}\right)^{2}\right)\left(z^{2}+\left(\frac{\sqrt{3} l}{2}\right)^{2}\right)} .
$$

The boundary conditions of this problem are that $a( \pm \infty)=l / 2, b( \pm \infty)=l \sqrt{3} / 2$, and $a(0)=0$. Following the general procedure of the first calculation, the free energy per unit length as a function of $a(h)$ and $b(h)$ was found to be:

$$
\begin{aligned}
f_{4}\{a(h), b(h)\}= & \sum_{i, j=0}^{4} c_{i j}^{(4)} a^{2 i} b^{2 j}+a^{2}\left(\frac{d a}{d h}\right)^{2} \sum_{i=0}^{2} u_{i} b^{2 i} \\
& +b^{2}\left(\frac{d b}{d h}\right)^{2} \sum_{i=0}^{2} v_{i} a^{2 i}+a b \frac{d a}{d h} \frac{d b}{d h} \sum_{i, j=0}^{1} w_{i j} a^{2 i} b^{2 j} .
\end{aligned}
$$

The coefficients $c_{i j}^{(4)}, u_{i}, v_{i}$, and $w_{i j}$ are given in Table \1. Again the first term can be viewed as a potential term due to the interaction between straight lines. Its form in the $a-b$ plane is shown in Fig 5. The other terms describe the tilt energies of the lines, with a fairly complex dependence on the positions of the lines. Looking at the form of the potential term $C_{4}(a, b)=\sum_{i, j=0}^{4} c_{i j}^{(4)} a^{2 i} b^{2 j}$ in Fig 5 we find that the interaction of straight lines in the LLL 


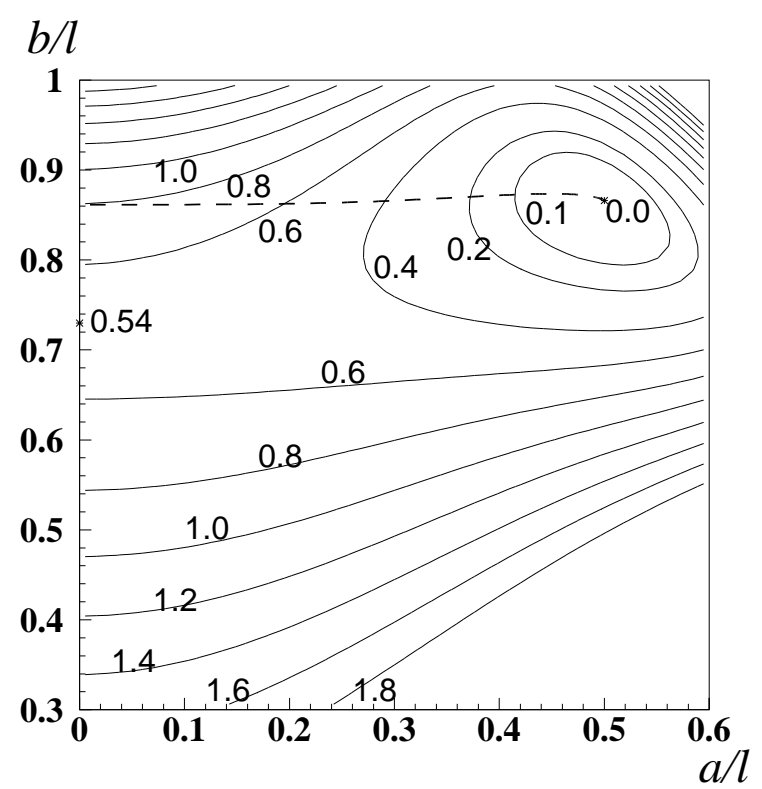

FIG. 5. The potential term, $C_{4}(a, b)=\sum_{i, j} c_{i j}^{(4)} a^{2 i} b^{2 j}$, for two lines crossing with the two nearest other lines also allowed to move. The dashed line shows the actual path taken in the full solution.

Abrikosov lattice is not at all as we might expect. One would think that parallel vortices always have a repulsive force between each other, but instead Fig 5 shows the surprising feature that if the two crossing lines are placed at the midpoint, the two nearest neighboring lines are actually pulled towards the midpoint! (i.e. the free energy is reduced by moving towards the center.) It is impossible to explain this effect by any simple two-body interaction between vortex-lines. The minimum value of $C_{4}(a, b)$ when $a=0$ is $C_{4}(0,0.73) \simeq 0.54$ which is considerably less than the value when the two extra lines remain at their original positions, $C_{4}(0, \sqrt{3} / 2) \simeq 0.79$. Therefore, allowing nearby lines to move will make a large difference to the potential terms for two lines crossing. However, one must also take into account the bending terms of all the lines involved in the full solution. Now, to find the configuration of $a(h)$ and $b(h)$ that minimizes the free energy cost of two lines crossing, we have to solve two coupled Euler-Lagrange equations of the form:

$$
\frac{\partial f_{4}}{\partial x_{i}}-\frac{d}{d h}\left(\frac{\partial f_{4}}{\partial x_{i}^{\prime}}\right)=0
$$

Substituting (3.12) into (3.13) leads to two coupled equations that were solved numerically with a relaxation method, using a software package. The resulting configuration from the solution to (3.13) is only slightly different from the configuration when the nearby lines are kept fixed. The two nearest lines move in by $\sim 0.03 l$ at $h=0$ (the actual path is shown in Fig 5). The difference in energy is very small, with the same answer to the accuracy quoted as (3.8). As we had expected the movement of the nearest lines to those crossing to affect the free energy change the most, it seems that the initial approximation of not allowing any of the surrounding lines to move is quite good, and we are fairly confident that the result 
(3.8) is close to the exact answer. The reason that the relaxation of surrounding lines has so little effect on the crossing energy is that it costs too much free energy to bend these extra lines over the short distance along $h$ that crossing takes place, so that the minimum in the potential term is never reached. This is a consequence of having a lattice of lines rather than pancake vortices.

\section{Search for a Two Line Braid}

The other extension for the two-line problem is not to restrict the lines to moving in one direction only but to allow for the possibility that the two lines may 'braid' around each other (by braid it is meant that the pair of lines twist around each other by half a rotation as we move along the direction of the lines). One of the original motivations to calculating the crossing energy was that it was thought to be an energy barrier to two topologically distinct configurations. For example, if one considers the two distinct braid configurations consisting of either a twist by $+\pi$ or by $-\pi$, then to go between the two states one has to pass through a configuration with two lines passing through each other. In fact, we show that there are no stable two-line braid-defects of the LLL Abrikosov solution (although the two-line crossing still remains an important energy barrier between larger entanglements).

We again allow the two neighboring lines to move symmetrically with respect to each other, but throughout the $x$ - $y$ plane. The positions of the two lines are given by $(X(h), Y(h))$ and $(-X(h),-Y(h))$ respectively (see Fig 6). The new order parameter is given by

$$
\psi_{2}(x, y, h)=\psi_{0}(x, y) \frac{\left(z^{2}-(X+i Y)^{2}\right)}{\left(z^{2}-\left(\frac{l}{2}\right)^{2}\right)}
$$

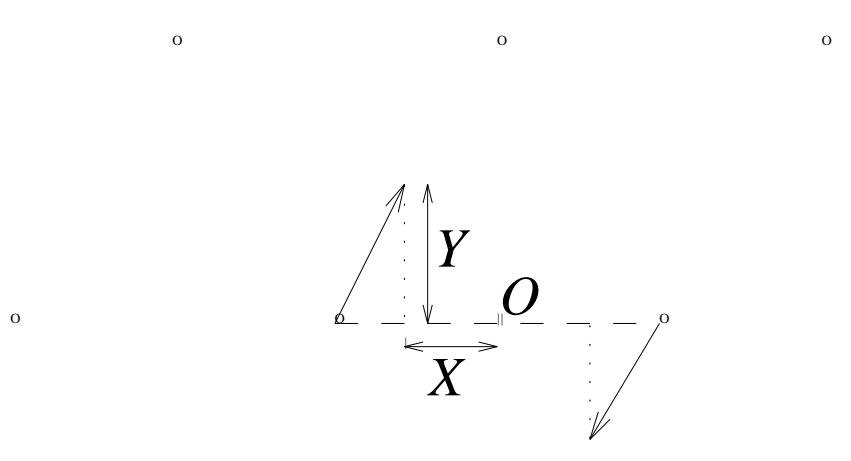

FIG. 6. Cross-section of the vortex-lattice with two lines allowed to braid around each other.

The boundary conditions for a braid are that $X(\infty)=l / 2, X(-\infty)=-l / 2, Y( \pm \infty)=0$. With a similar calculation to the one parameter case, the free energy as a function of $X$ and $Y$ can be derived: 


$$
f_{2}\{X(h), Y(h)\}=\sum_{i, j=0}^{4} c_{i j}^{(2)} X^{2 i} Y^{2 j}+c^{(2)}\left(X^{2}+Y^{2}\right)\left(\left(\frac{d X}{d h}\right)^{2}+\left(\frac{d Y}{d h}\right)^{2}\right)
$$

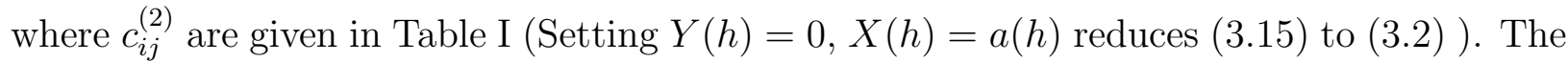
first term $C_{2}(X, Y)=\sum_{i, j=0}^{4} c_{i j}^{(2)} X^{2 i} Y^{2 j}$ is the interaction term without bending of the lines, shown in Fig [. We could now go on as before in trying to solve the Euler-Lagrange equations to minimize the integral of (3.15) subject to the braid boundary conditions. Inspection of Fig 0 reveals that there is no point in this- there can be no braid solution for this case! This is because the form of the potential part of the free energy is always increasing as we increase $Y$ away from the midpoint (the midpoint is a saddle-point of $C_{2}(X, Y)$ ). As the bending term will increase with $\left(X^{2}+Y^{2}\right)$ for any given tilt, it is clear that the free energy cost of any two line braid will increase continuously as the path of the braid moves away from the midpoint. i.e. there are no stationary configurations for the braid boundary conditions other than the direct crossing already discussed.

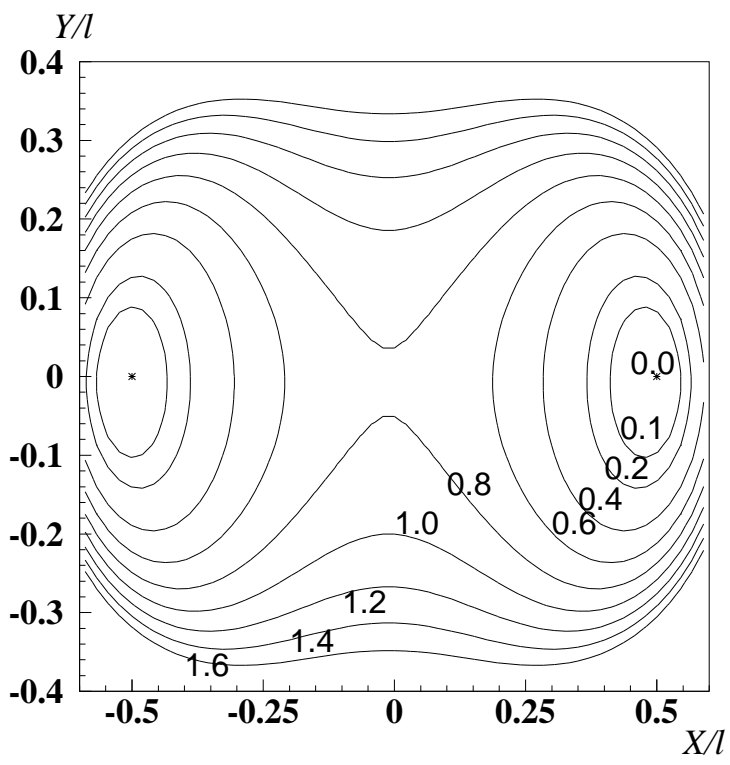

FIG. 7. The potential term, $C_{2}=\sum_{i, j} c_{i j}^{(2)} X^{2 i} Y^{2 j}$, for two lines allowed to braid or cross. A braid will correspond to going from the minimum at $(0.5,0)$ around to the minimum at $(-0.5,0)$. The path with lowest potential will be along the $X$-axis, which corresponds to a crossing defect.

One might find it extremely surprising that two straight vortices placed at the same point will not repel each other along the $Y$ direction as well as the $X$ direction, even in the presence of the surrounding lattice. This seems to be another non-intuitive feature of the way vortices interact in the LLL. It is a possibility that the absence of a braid solution is an artefact of our approximation of not allowing the surrounding vortices to move. However, the result of Section एIIB suggests that these surrounding lines should not have a great effect on the two-line defect. Also, a similar calculation to that in Section [ITB was performed where the two nearest neighboring lines were allowed to move in response to the motion 
of the two lines along $Y$. This still gave the same result that the potential term always increases as $Y$ increases.

\section{Three- and Six-Line Defects}

Due to the symmetries of the triangular lattice, there are two other forms of braid defects which can be handled in the same way as for the two-line defect. Allowing motion of the three lines that form the smallest triangle (see Fig 8), or the six lines that form a hexagon surrounding one central line (Fig 9). If we assume that in the lowest energy configurations

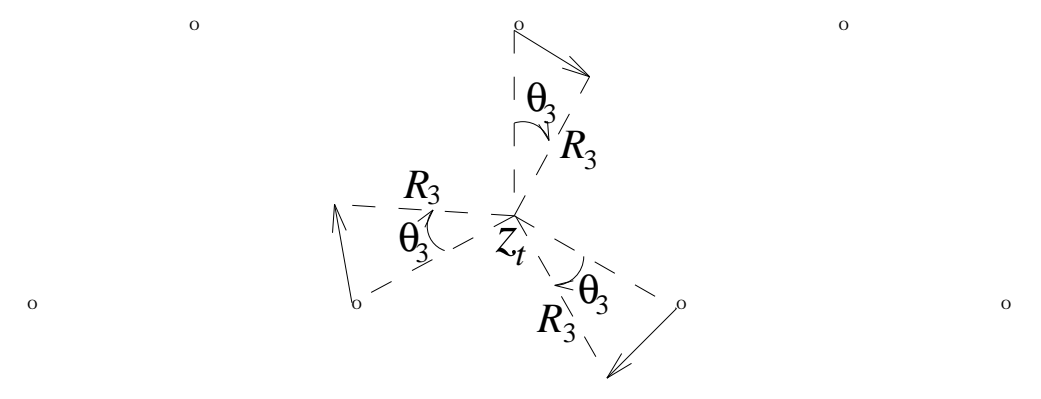

FIG. 8. Cross-section of the vortex-lattice with three lines allowed to braid around each other. The center of the defect is labelled $z_{t}$.

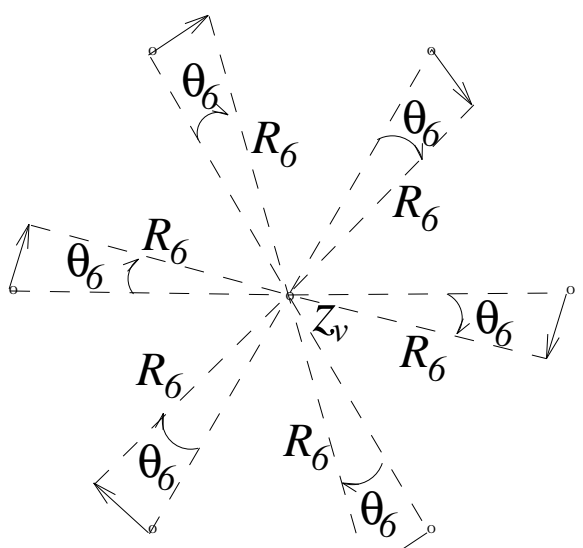

FIG. 9. Cross-section of the vortex-lattice with six lines allowed to braid around each other. The position of the central vortex is $z_{v}$.

the lines in these defects move symmetrically about their center, we can write the order parameter of the defects as: 


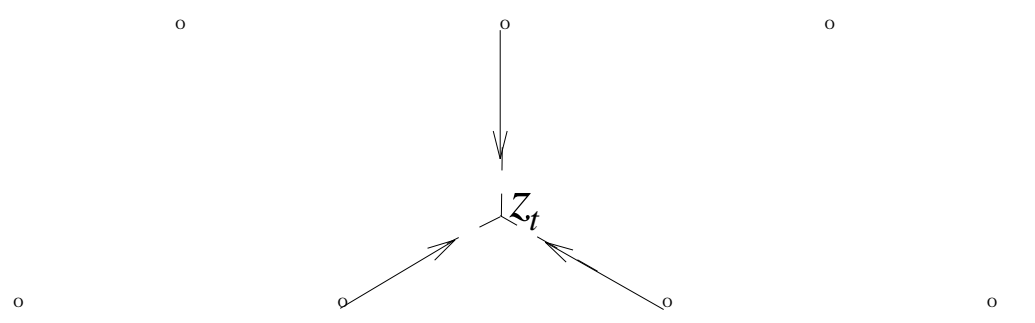

FIG. 10. Cross-section of the vortex-lattice showing the path taken in a three line crossing defect.

$$
\begin{aligned}
& \psi_{3}(x, y, h)=\psi_{0}(x, y) \frac{\left(\left(z-z_{t}\right)^{3}+i R_{3}{ }^{3} e^{i 3 \theta_{3}}\right)}{\left(\left(z-z_{t}\right)^{3}+i\left(\frac{l}{\sqrt{3}}\right)^{3}\right)} \\
& \psi_{6}(x, y, h)=\psi_{0}(x, y) \frac{\left(\left(z-z_{v}\right)^{6}-R_{6}{ }^{6} e^{i 6 \theta_{6}}\right)}{\left(\left(z-z_{v}\right)^{6}-l^{6}\right)}
\end{aligned}
$$

The free energy costs per unit length are of the form:

$$
f_{n}\left\{R_{n}(h), \theta_{n}(h)\right\}=\sum_{i, j, k} c_{i j k}^{(n)} R_{n}^{3 i} \cos ^{j} n \theta_{n} \sin ^{k} n \theta_{n}+c^{(n)}\left(R_{n}^{2 n-2}\left(\frac{d R_{n}}{d h}\right)^{2}+R_{n}^{2 n}\left(\frac{d \theta_{n}}{d h}\right)^{2}\right)
$$

with $n=3,6$. The constants $c_{i j k}^{(3)}$ and $c_{i j k}^{(6)}$ are given in Table III. $c^{(3)} \simeq 317.9 / l^{6}$, and $c^{(6)} \simeq 117.7 / l^{12}$. The conditions for a braid are $\theta_{n}(\infty)=0, \theta_{n}(-\infty)=2 \pi / n$ and $R_{n}( \pm \infty)$ equal to the ground state value. Again, to minimize the total free energy of given defects, two coupled E-L equations must be satisfied. The results of the numerical solutions to the E-L equations are: (i) There is no braid solution for the three line defect, other than where the three lines meet at the center of the triangle (as in Fig 10). (ii) The free energy cost of the three lines 'crossing' is lower than the cost of two lines crossing, the result being:

$$
\Delta F_{3 \times}=0 \cdot 51 k_{B} T\left|\alpha_{T}\right|^{\frac{3}{2}} .
$$

(iii) There is a solution for a six-line braid with an energy cost of:

$$
\Delta F_{6 b r}=2 \cdot 19 k_{B} T\left|\alpha_{T}\right|^{\frac{3}{2}} .
$$

(iv) There is also a solution for the six-line defect where the lines all meet at a point on the center line (see Fig 11), and this crossing defect has a lower free energy cost than the six-line braid: 


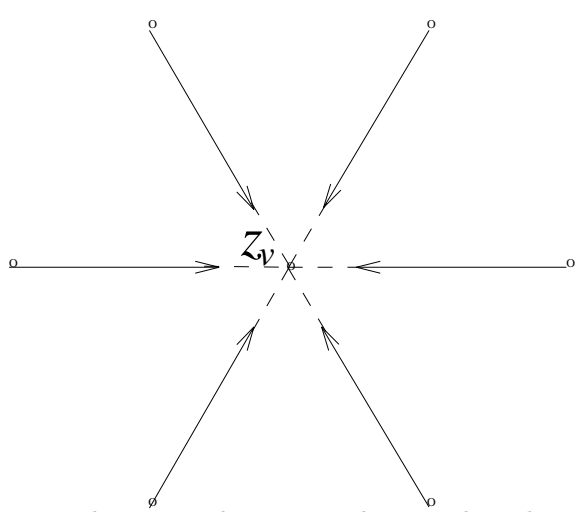

FIG. 11. Cross-section of the vortex-lattice showing the path taken in a six line crossing defect.

$$
\Delta F_{6 \times}=1 \cdot 38 k_{B} T\left|\alpha_{T}\right|^{\frac{3}{2}}
$$

It will seem surprising that we have found the energy for three lines to meet at a point to be lower than the energy for two lines. This is due to the potential term when three lines meet, $c_{000}^{(3)} \simeq 0.54$, being lower than the potential term for two lines at their midpoint, $c_{00}^{(2)} \simeq 0.79$. Note that both of these values are for when the surrounding lattice remains fixed, so it is quite possible that relaxation of the surrounding lattice will reverse this inequality in the potentials. As was shown in Section IIIB, allowing the surrounding lines to move may decrease the potential at the crossing point significantly. However, we also showed in Section IIIB that the full solution including the bending terms in the free energy does not allow the surrounding lines to relax much, and so we believe that this result of $\Delta F_{3 \times}<\Delta F_{2 \times}$ will hold even when all surrounding lines are taken into account.

\section{E. Discussion on Two-, Three- and Six-Line Braid Solutions}

To compare the calculations for the above defects, and to understand what is required for braid solutions to exist it is useful to cast the free energies (3.15, 3.18) into the same form. We do this by making the transformations, $\xi=(X+i Y)^{2}, \xi=R_{3}{ }^{3} \exp \left(i 3 \theta_{3}\right)$, or $\xi=R_{6}{ }^{6} \exp \left(i 6 \theta_{6}\right)$ respectively. This gives a free energy per unit length in terms of the complex variable $\xi$ :

$$
\begin{aligned}
f_{n}\{\xi(h)\} & =\sum_{i j=0}^{4} d_{i j}^{(n)}[\operatorname{Re}(\xi)]^{i}[\operatorname{Im}(\xi)]^{j}+d^{(n)}\left(\left(\frac{d \operatorname{Re}(\xi)}{d h}\right)^{2}+\left(\frac{d \operatorname{Im}(\xi)}{d h}\right)^{2}\right) \\
& =D_{n}(\xi)+d^{(n)}\left|\frac{d \xi}{d h}\right|^{2},
\end{aligned}
$$

for $n=2,3,6$. With this substitution, the two- three- and six-line braids all have the same boundary conditions: $\xi( \pm \infty)=x_{n}$ where $x_{n}$ is real, and $\xi$ goes around the origin of the complex plane once, as $h$ goes from $-\infty$ to $+\infty . x_{2}=(l / 2)^{2}, x_{3}=(\sqrt{3} l / 2)^{3}$, and $x_{6}=l^{6}$. In each case, $\xi=x_{n}$ corresponds to the only minimum of $D_{n}(\xi)$ (the ground 
state configuration). In the analogy with classical dynamics, the solutions to these boundary conditions which minimize the integral over $h$ of (3.23) correspond to the motion of a particle of mass $m=2 d^{(n)}$ in a two-dimensional potential $V(x, y)=-D_{n}(x+i y)$.

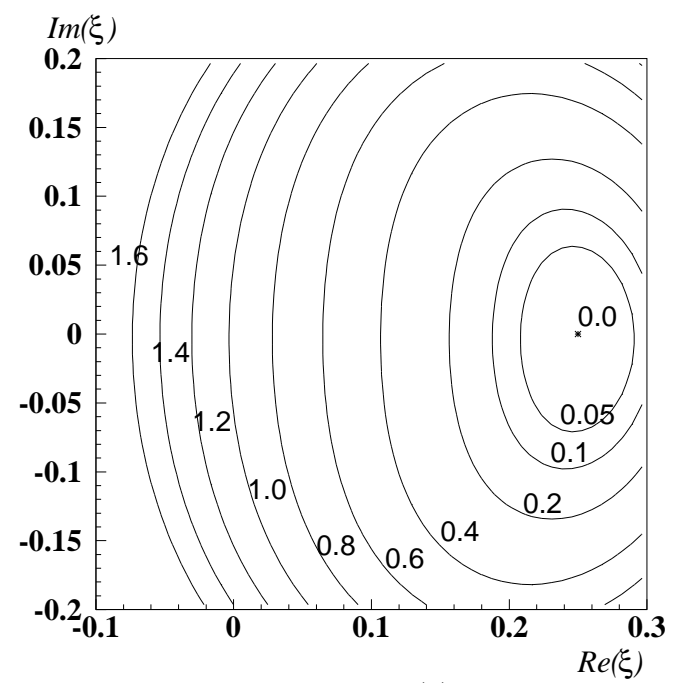

FIG. 12. The potential term, $D_{2}(\xi)=\sum_{i, j} d_{i j}^{(2)}[\operatorname{Re}(\xi)]^{i}[\operatorname{Im}(\xi)]^{j}$, where $\xi=(X+i Y)^{2}$. A two-line braid corresponds to starting and ending at the minimum at $(0.25,0)$ while going around the origin once. The path with the lowest potential will correspond to a crossing defect.

It is clear that a braid solution where the lines in the defect do not meet at their center will only exist if there is a 'valley path' in the $\xi$-plane for the function $D_{n}(\xi)$ which goes around the origin, starting and finishing at $\xi=x_{n}$. Otherwise any braid configuration will be able to continuously lower its total free energy by allowing $\xi$ to move nearer the origin as it goes around and the only solution will be a crossing defect (where $\xi$ goes to the origin and back along the real axis). By looking at the forms of $D_{n}(\xi)$ in Figs 12, 13 and 14 one can see why there is no braid solution for $n=2,3$, as there are no valley paths. For $n=6$ the presence of a braid solution is explained by the almost circular valley path in $D_{6}$.

The presence of a valley path is a minimum requirement for a braid solution to exist. It does not guarantee that this braid will have a lower energy cost than the corresponding crossing defect, as our results for $n=6$ show. Even though the height of the potential term $D_{6}(\xi)$ is higher at the origin than at any point in the braid path, the six-line defect can still lower its total free energy when $\xi$ goes to the origin and back more than when $\xi$ goes around the valley as the bending term in (3.23) will be lower when there is less of a change in $|\xi|$. For the braid boundary conditions, this means that for a defect of given length along $h$, the bending term will be lower when the length of the path in the $\xi$ plane is smaller. Thus there is a balance here between the potential term which favours the braid defect around the valley, and the bending term which favours the crossing defect. In this case the relative terms give the lower total free energy to the crossing defect, although stationary solutions exist for both defects with a free energy barrier between the two. 


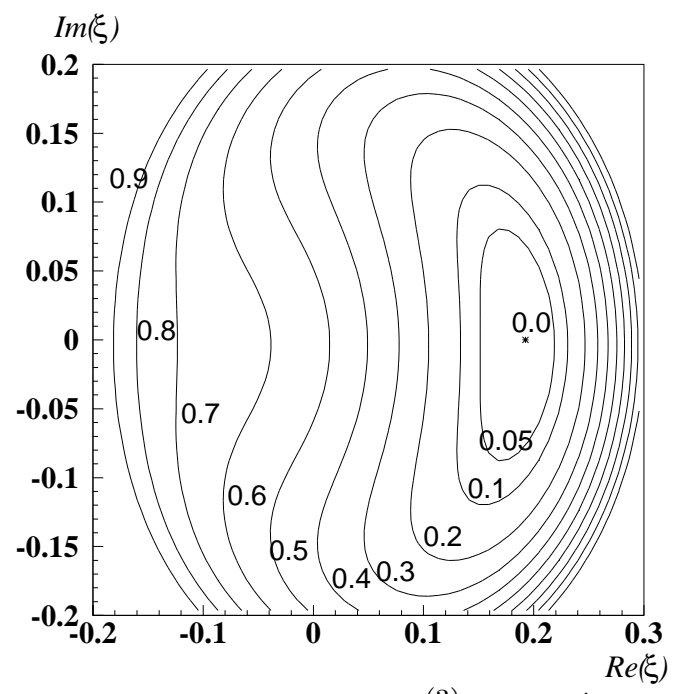

FIG. 13. The potential term, $D_{3}(\xi)=\sum_{i, j} d_{i j}^{(3)}[R e(\xi)]^{i}[\operatorname{Im}(\xi)]^{j}$, where $\xi=R_{3}{ }^{3} \exp (i 3 \theta)$. Again, the three-line braid path with the lowest potential will correspond to a crossing defect.

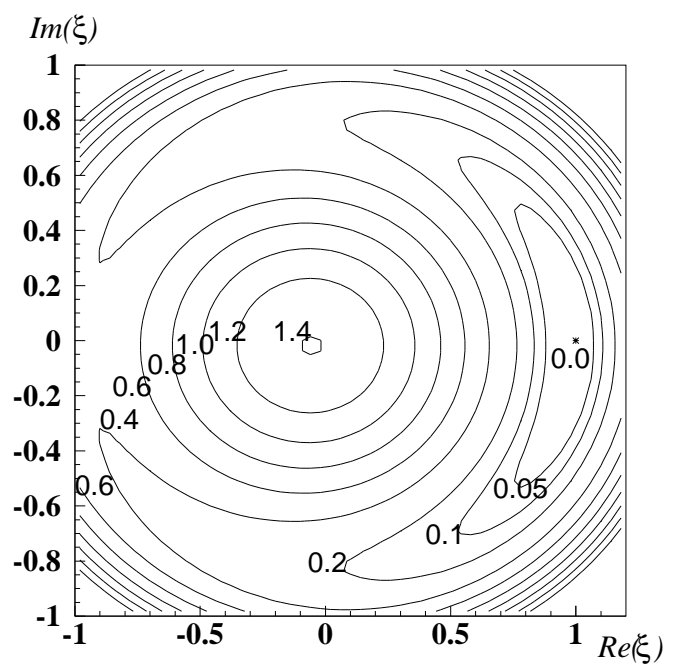

FIG. 14. The potential term, $D_{6}(\xi)=\sum_{i, j} d_{i j}^{(6)}[R e(\xi)]^{i}[\operatorname{Im}(\xi)]^{j}$, where $\xi=R_{6}{ }^{6} \exp (i 6 \theta)$. Now there is a 'valley path' in the potential that corresponds to a braid without the lines meeting at the center. 


\section{F. The Twelve-Line Braid Defect}

So far in this paper, the symmetry of the configurations has allowed us to reduce the problems to just two coupled equations in two parameters, describing the two-dimensional motion of a single 'particle'. In order to consider larger braids than the 2- 3- and 6-line defects, one now has to include more degrees of freedom. The simplest problem we can think of with more than six-lines involved is the 'twelve line hexagonal defect' (see Fig 15). We can still take advantage of the symmetry of this configuration by assuming that it always costs least free energy when the six lines on the vertices of the hexagon move symmetrically about the hexagon's center, and the six lines on the sides of the hexagon also move symmetrically. This allows us to reduce the number of degrees of freedom in this defect to four (corresponding to the two-dimensional motion of two particles in the E-L equations).

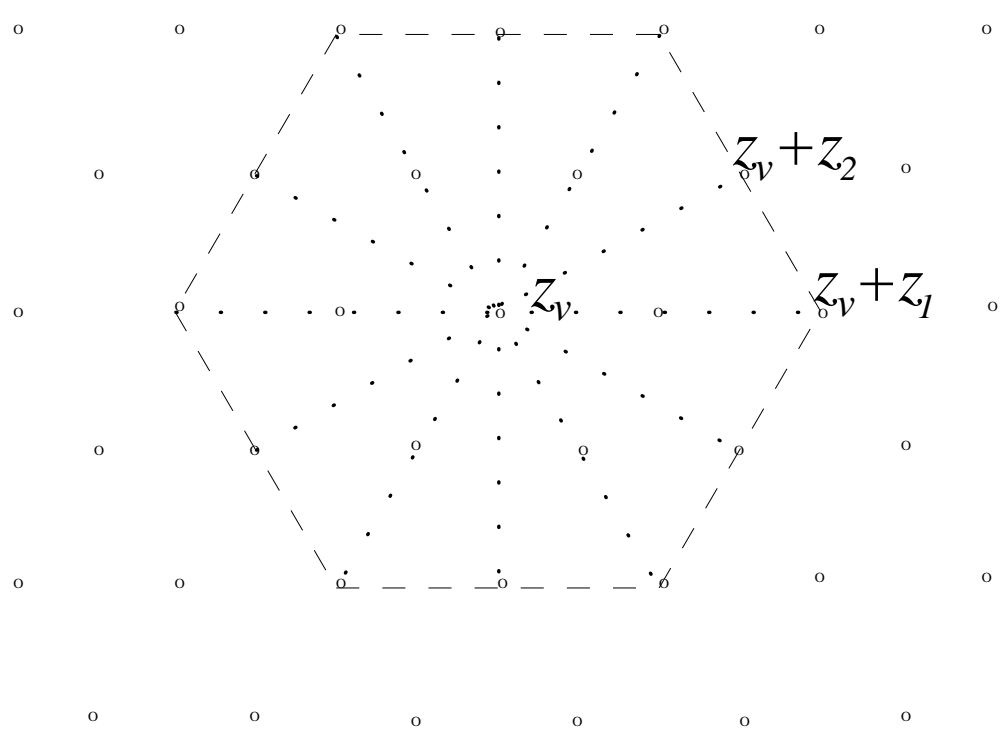

FIG. 15. Cross-section of the vortex-lattice with twelve lines allowed to move within a defect. The dashed line shows a possible braid path. The dotted line shows the path of a crossing defect.

In the ground state, the complex positions of the 'edge lines' are given by the roots of $\left(z-z_{v}\right)^{6}-z_{1}^{6}=0$, and the 'corner lines' by $\left(z-z_{v}\right)^{6}-z_{2}^{6}=0$, where $z_{1}=2 l$ and $z_{2}=\sqrt{3} l \exp (i \pi / 6)$. If the complex positions relative to $z_{v}$ move from $\left(z_{1}, z_{2}\right)$ to the new positions $\left(\zeta_{1}, \zeta_{2}\right)$ then the order parameter of the system can be written:

$$
\psi_{12}(x, y, h)=\psi_{0}(x, y) \frac{\left(\left(z-z_{v}\right)^{6}-\zeta_{1}^{6}\right)\left(\left(z-z_{v}\right)^{6}-\zeta_{2}{ }^{6}\right)}{\left(\left(z-z_{v}\right)^{6}-z_{1}^{6}\right)\left(\left(z-z_{v}\right)^{6}-z_{2}{ }^{6}\right)} .
$$

To find the free energy of twelve-line defects, the same procedure is followed where we substitute into (2.13), then integrate over the $x$ - $y$ plane, to get a free energy per unit length as a function of the complex $\zeta_{1}(h)$ and $\zeta_{2}(h)$. We omit the details of the calculation, which requires a lot of space but results in four coupled non-linear second order equations in the 
real and imaginary parts of $\zeta_{1}{ }^{6}$ and $\zeta_{2}{ }^{6}$. This system of four E-L equations was numerically solved subject to the braid boundary conditions. The actual path of the braid of lowest free energy was close to the hexagonal perimeter, and this solution gave a total free energy cost of:

$$
\Delta F_{12 b r} \simeq 6 \cdot 5 k_{B} T\left|\alpha_{T}\right|^{\frac{3}{2}}
$$

A crossing solution was also looked for, where all twelve lines meet at a point on the central line (at $z=z_{v}$ ), moving along the dotted line in Fig 15. For the six line case this form of defect has a lower energy cost than a braid. For twelve lines crossing the result is

$$
\Delta F_{12 \times} \simeq 8 \cdot 6 k_{B} T\left|\alpha_{T}\right|^{\frac{3}{2}}
$$

This is higher than the twelve line braid free energy. We expect that all larger braids will have lower energies than the corresponding crossing defects.

\section{CALCULATIONS FOR LARGE DEFECTS}

In this section, the energy cost is calculated for an infinite straight screw dislocation (see Fig (16), and also for two opposite screw dislocations a large distance apart. The results of these are used to give the energy cost for very large braids. The single screw dislocation may be a first step towards calculating the energy costs of the screw-edge dislocation loops that are thought to be important in describing the dynamics of the Abrikosov crystal 2,13 . The creation/growth of large braid defects has been proposed as a mechanism for the longitudinal resistivity in type-II superconductors 14.15 .

\section{A. Infinite Screw Dislocation}

The order parameter of the infinite screw defect in Fig 16 will depend on only one free parameter, $s(h)$ :

$$
\psi_{s}(x, y, h)=\psi_{0}(x, y) \frac{\sin \pi(z-s(h))}{\sin \pi z}
$$

where the origin of the $x-y$ plane is now taken to be at the ground state position of one of the lines involved in this defect. This order parameter replaces the first order zeros of $\psi_{0}$ at $z=n l$ with first order zeros at $z=n l+s(h)$.

The usual procedure is now followed of integrating the difference of the free energy density over the $x-y$ plane. All of the integrands are periodic in $x$, giving terms which all diverge linearly with the system size in the $x$-direction, $L_{x}$. Also, the bending term contains an integral which tends to a constant for large $y$, while all other terms are convergent when integrated over $y$. This leads to the result for the free energy per unit length as a function of $s(h)$ :

$$
f_{s}\{s(h)\}=\sum_{i=1}^{2} \frac{L_{x}}{l} c_{i}^{(s)} \sin ^{2 i}\left(\frac{\pi s}{l}\right)+\frac{L_{x} L_{y}}{l^{2}} c^{(s)}\left(\frac{d s}{d h}\right)^{2}
$$



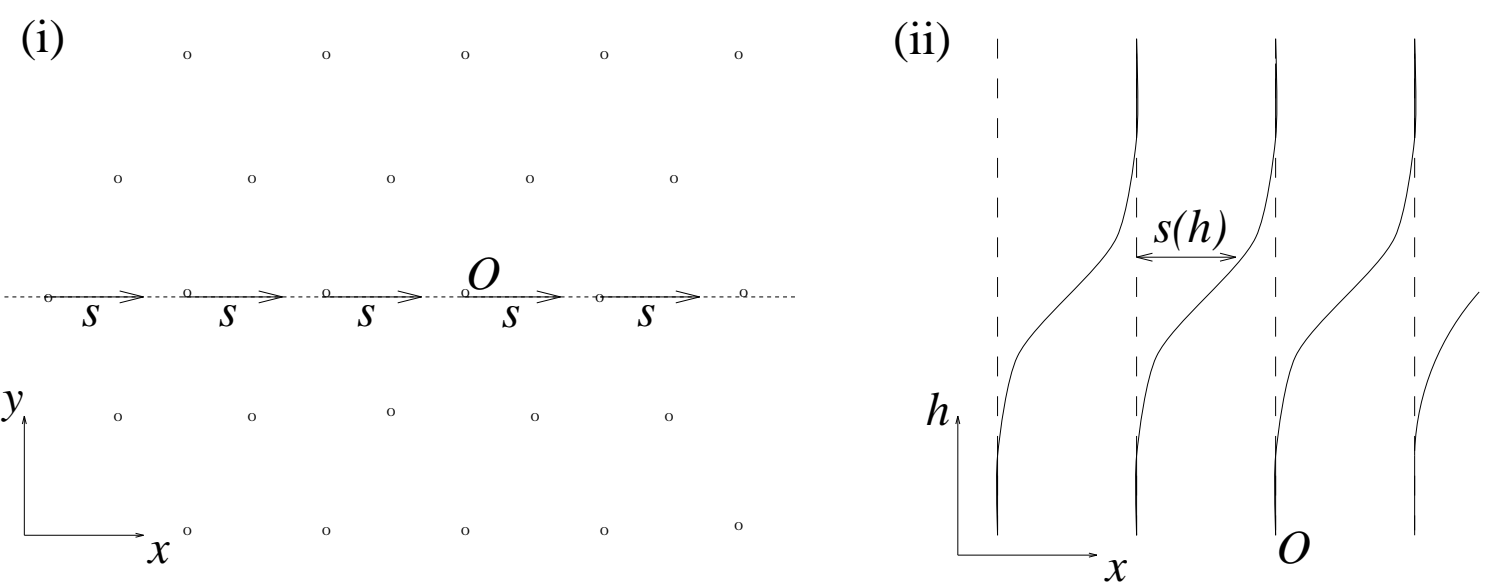

FIG. 16. (i) Cross-section of the lattice in the $x-y$ plane showing the path of an infinite straight screw defect. (ii) Schematic side-view of the same defect.

with $c_{1}^{(s)} \simeq 0.2883, c_{2}^{(s)} \simeq 2.288 \times 10^{-4}$ and $c^{(s)}=\pi^{2}\left\langle\left|\psi_{0}\right|^{2}\right\rangle \simeq 9.8696$. To find the general form of $s(h)$ that minimizes the integral over $h$ of (4.2), for any given system size, we make the substitution $h \rightarrow \tilde{h}=h / \xi_{s}$ with $\xi_{s}=\sqrt{L_{y} / l}$. This gives a free energy density as a function of $s(\tilde{h})$ with no dependence on $L_{y}$ and a linear dependence on $L_{x}$. The resulting E-L equation in $s(\tilde{h})$ is independent of $L_{x}$ and $L_{y}$, so increasing $L_{y}$ increases the scale of the screw defect along the $h$-direction, $\xi_{s}$, and the resulting free energy cost (found by integrating $f_{s}\{s(\tilde{h})\}$ over all $\tilde{h}$ with $s(\tilde{h})$ the solution to the E-L equation), is proportional to $L_{x} \sqrt{L_{y}}$ :

$$
\Delta F_{s} \simeq 0 \cdot 54 \frac{L_{x} \sqrt{L_{y}}}{l^{3 / 2}} k_{B} T\left|\alpha_{T}\right|^{\frac{3}{2}}
$$

That is, the free energy cost increases linearly with the number of lines involved in the defect $\left(n \propto L_{x}\right)$ as one would expect, but it also has a divergence as the size of the system increases in the direction perpendicular to the defect. This is yet another result that is a special feature of the long-range vortex-vortex interactions in the lowest Landau level, and one that would not occur with short-range two-body interactions as in the London limit. The long range dependence of the bending term in the screw defect has important implications to the free energy cost of large braids, which are developed in the next section.

\section{B. Two Opposite Screws}

A similar calculation which removes this extra divergence of the energy with $L_{y}$ is of two 'opposite' screws a large distance $W_{y}=n \sqrt{3} l$ from each other. This defect has the order parameter:

$$
\psi_{2 s}(x, y, h)=\psi_{0}(x, y) \frac{\sin \pi\left(z-\frac{n l \sqrt{3}}{2} i-s(h)\right) \sin \pi\left(z+\frac{n l \sqrt{3}}{2} i+s(h)\right)}{\sin \pi\left(z-\frac{n l \sqrt{3}}{2} i\right) \sin \pi\left(z+\frac{n l \sqrt{3}}{2} i\right)},
$$


where the origin in $(x, y)$ is now at a vortex half-way between the two opposite screws. For large enough $n$, the potential terms in the free energy cost for a given $s(h)$ become just twice the corresponding terms for the single screw. The bending term now contains an integral that diverges linearly with $n$ rather than $L_{y}$. The actual result is

$$
f_{2 s}\{s(h)\}=\sum_{i=1}^{2} 2 \frac{L_{x}}{l} c_{i}^{(s)} \sin ^{2 i}\left(\frac{\pi s}{l}\right)+4 n \sqrt{3} \frac{L_{x}}{l} c^{(s)}\left(\frac{d s}{d h}\right)^{2} .
$$

To solve the resulting E-L equation we make a similar transformation as before: $h \rightarrow$ $\tilde{h}=(2 n \sqrt{3})^{1 / 2}$. This leads to the same E-L equation as for a single screw, and the free energy cost of this 'double screw' is:

$$
\Delta F_{2 s} \simeq 1 \cdot 5 \frac{L_{x} \sqrt{W_{y}}}{l^{3 / 2}} k_{B} T\left|\alpha_{T}\right|^{\frac{3}{2}} .
$$

As might be deduced from the result for the single screw, the energy of a large double screw is proportional to the square root of the distance between the two screws. It also suggests that, at least where the LLL approximation is valid, the energy cost of large braids will not simply be proportional to the circumference of the braids (or equivalently the radius of, or the number of lines in the braid) as has often been suggested. This is shown in the next section.

\section{A Limiting Form For Large Braids}

We now show that as a braid of general shape becomes very large, the free energy cost has a simple form depending only on the area enclosed by the braid and the length of the perimeter of the defect. We consider a large braid involving $n$ vortices (an example would be the braid in Fig 17), where the lines move from $z=z_{i}$ to $z=z_{i+1}$ as $h$ increases between $\pm \infty$, with $i=1, n$ and $z_{n+1}=z_{1} \exp (2 i \pi)$.

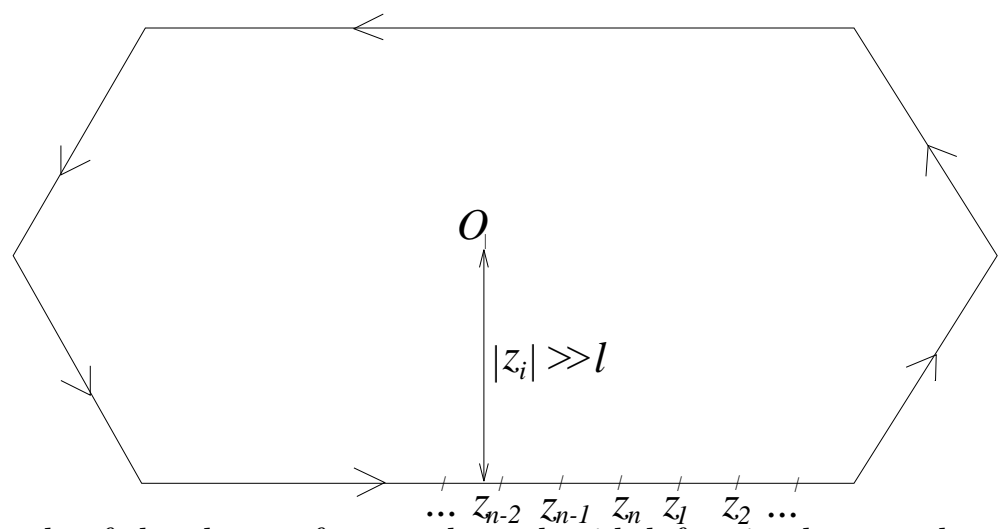

FIG. 17. Example of the shape of a very large braid defect in the $x-y$ plane where the vortices move from $z_{i}$ to $z_{i+1}$ with increasing $h$.

Three assumptions are required to make the necessary approximations: (i) The regions over which the braid is straight are much larger than regions where the effect of the 'corners' 
of the braid on the potential term are important. (ii) All lines in the braid move by the same distance, $s(h)$ towards their nearest neighbor. i.e. $\zeta_{i}(h)=z_{i}+\left(z_{i+1}-z_{i}\right)(s(h) / l)$. (iii) The braid is everywhere a large distance from the 'center' of the braid. The center, which is where we place the origin, $O$, is defined by $O=(1 / n) \sum z_{i}$. Therefore this condition means that $\left|z_{i}\right| \gg l$. These assumptions allow us to approximate the potential term in the free energy cost using the result for a straight screw (which is proportional to the length of the screw) and to calculate the bending term in a simple form as follows.

The order parameter of this general $n$-line braid is given by

$$
\psi_{n b r}(x, y, h)=\psi_{0}(x, y) \frac{\prod_{i=1}^{n} z-z_{i}-\left(z_{i+1}-z_{i}\right)(s(h) / l)}{\prod_{i=1}^{n} z-z_{i}},
$$

To find the bending term we need the partial derivative of the order parameter with respect to $s$ :

$$
\begin{aligned}
\frac{\partial \psi_{n b r}}{\partial s} & =\psi_{0} \frac{\sum_{j=1}^{n}\left\{-\left(z_{j+1}-z_{j}\right)(1 / l) \prod_{i=1, i \neq j}^{n}\left(z-z_{i}-\left(z_{i+1}-z_{i}\right)(s(h) / l)\right)\right\}}{\prod_{i=1}^{n}\left(z-z_{i}\right)} \\
& \simeq \psi_{0} \sum_{j=1}^{n} \frac{-\left(z_{j+1}-z_{j}\right) / l}{z-z_{j}},
\end{aligned}
$$

where the second line uses condition (iii). We now consider this sum when the complex coordinates $z=x+i y$ are far from the perimeter of the braid. That is, when $\left|z-z_{j}\right| \gg 1$ for all $j$, the sum becomes

$$
\begin{aligned}
\frac{\partial \psi_{n b r}}{\partial s} & =\sum_{j=1}^{n} \psi_{0} \ln \left(\frac{z-z_{j+1}}{z-z_{j}}\right) \\
& = \begin{cases}2 i \pi \psi_{0} & \text { if } z \text { inside braid. } \\
0 & \text { if } z \text { outside braid. }\end{cases}
\end{aligned}
$$

That is, the partial derivative of the order parameter is periodic within the braid and zero outside. Although this result does not hold when $z$ is near the perimeter, when we integrate over the $x-y$ plane then for large braids the integral will be dominated by the contribution where this does hold. The limiting result for the bending term is:

$$
\int \frac{d^{2} r}{l^{2}}\left|\frac{\partial \psi_{n}}{\partial h}\right|^{2}=\frac{A}{l^{2}} \pi^{2}\left\langle\left|\psi_{0}\right|^{2}\right\rangle\left(\frac{d s}{d h}\right)^{2}
$$

where $A$ is the area enclosed by the braid. Adding the potential term taken from the infinite screw calculation gives a free energy cost for the large braid as a function of $s(h)$ as:

$$
f_{n b r}\{s(h)\}=\sum_{i=1}^{2} \frac{P}{l} c_{i}^{(s)} \sin ^{2 i}\left(\frac{\pi s}{l}\right)+4 \frac{A}{l^{2}} c^{(s)}\left(\frac{d s}{d h}\right)^{2},
$$

with $P$ the length of the perimeter of the braid. Making the correct transformation in the length scale of the defect, $h \rightarrow \tilde{h}=h \sqrt{P l / 4 A}$ allows us to use the results from Section $\mathbb{E A}$ to find a total free energy cost for a large braid of: 


$$
\Delta F_{n b r} \simeq 1 \cdot 1 \frac{\sqrt{P A}}{l^{\frac{3}{2}}} k_{B} T\left|\alpha_{T}\right|^{\frac{3}{2}} .
$$

An important caveat for the large defects in this section is that our results will only be good for defects of less than a certain size. As in all of our calculations we have used the LLL approximation, we have assumed that we may ignore fluctuations in the microscopic flux density. This will lead to good approximations of the energy costs only when the size of the defects is less than the distance over which the flux density can change significantly, which is the magnetic penetration depth. In the $x-y$ plane, the dimensions of the defect must be less than $\lambda_{a b}$. The extent of the defect in the field direction must be less than $\lambda_{c}$.

\section{APPLICATIONS}

\section{A. The nature of the vortex state}

The consequences of finding no stable two-line (or three-line) braids, and of a relatively high free energy cost for the six-line braid greatly affects our picture of the vortex state in thermal equilibrium at and above the irreversibility line. If stable two-line braids of low free energy existed they would be created in large numbers by thermal fluctuations at relatively low temperatures, and give rise to a system with vortices twisting around each other in the lattice, i.e. an entangled state. However, the absence of such low energy braids means that extensive entanglement will not occur over a large region of the $H$ - $T$ phase diagram that includes the irreversibility line, as we now show.

We deduce from our results that the braid defect of lowest free energy is the six-line hexagonal braid, with an energy cost given in Eq. (3.20). This is because we have shown that smaller braids have no saddle point solutions and the lines can just pull through each other while continuously lowering their free energy. We use the result (3.20) to make an estimate of how many of these braids will be present in a sample of superconducting material of dimensions typical to those available. The stable six-line braid defect extends along the direction of the vortices a distance $L_{6 b r} \sim 50 \xi_{c}$, where $\xi_{c}$ is the superconducting coherence length along the field direction. If we consider a sample with dimensions along the field axis of $L_{c}$, then in thermal equilibrium, the average number of braids along any six lines that surround a given line will be $N_{6 b r}=\left(L_{c} / L_{6 b r}\right) \exp \left(-\Delta F_{6 b r} / k_{B} T\right)$. The number of six-line braids that any one vortex line is involved in will be $6 N_{6} \mathrm{br}$.

If we take an estimate of $\alpha_{T}$ on the melting line as $\alpha_{T} \sim-8$ we find that the exponential gives a factor of $\exp \left(-\Delta F_{6 b r} / k_{B} T\right) \sim e^{-50} \sim 10^{-22}$. Taking an extreme lower limit on the coherence length of high- $T_{c}$ superconductors of $\xi_{c} \sim 10^{-10} \mathrm{~m}$, we would need a sample size of $L_{c} \simeq 10^{13} \mathrm{~m}$ for there to be an average of one braid defect on any given line! Alternatively, if we consider the number of six-line braids in the whole sample, then at a field of $10 \mathrm{~T}$ there will be approximately one braid defect in $10^{5} \mathrm{~mm}^{3}$ at the melting line. (Decreasing the external field reduces the number of defects if we remain on the same $\alpha_{T}=$ const line).

We now consider at what regimes the braid defects will proliferate. Using the above estimates we find that, for a sample with $L_{c} \sim 1 \mathrm{~mm}$ there will be an average one braid defect per line at $\alpha_{T} \sim-3$. Above this line in the $H$-T phase diagram, braid defects will be plentiful and the vortex state will be strongly entangled. Below this line however, the average 
line will not be involved in such defects and the vortices can be well identified between the top and the bottom of the sample. These arguments make it clear that below the melting line in the crystalline phase, and for a large region above in the flux-liquid phase, the vortices in type-II superconductors of sizes currently available will not be in a strongly entangled state, when at thermal equilibrium. This is contrary to the picture previously presented in $\mathrm{l}$ where entanglement has been claimed to play a major role in these systems near the irreversibility line. Instead, the lines maintain a correlation between their positions at the top and and their positions at the bottom of the sample (for a recent experimental realization, sed16). Note that this does not mean the lines are not moving, either by fluctuations transverse to their length, or their motion as a whole when in the liquid state. It simply means that the lines do not twist around each other to form long-lived topologically distinct entangled states.

Of course, our results are only strictly valid in the high field regime where the LLL approximation holds. However, if braid defects are of such high energy costs here, it is hard to see how the Boltzmann factors will come down as we lower the temperature or field. Also our calculations are within the low-temperature crystalline state and it may be questioned if we can extend the results to the liquid state. These defects depend only locally on the surrounding lattice, and we expect the liquid state to retain crystalline order over such length scales for a large region above melting, in which case our results may still apply. It is also important to note that the absence of entanglement is a size dependent phenomenon. In a big enough sample, entanglements will arise in principle, but, at least near the melting line, they will be completely absent in practice. The above arguments are only for a system in thermal equilibrium, and if the system has been rapidly cooled from a high temperature to near the melting line it is still possible for entanglements to be present for some time.

The absence of any long lived braid defects within the system of vortices can be tested experimentally. Neutron scattering, or possibly muon spin resonance, could be used to investigate the magnetic fields associated with the braids which are transverse to the externally applied field. There should be a locally qualitative difference between the resulting spectra due to the fluctuations in the transverse displacement of a vortex along its length, and from the local transverse field of a stable braid defect.

\section{B. Longitudinal Resistivity due to Braid Defects}

Experiments have been performed where a current is applied between terminals on the top and bottom faces of a superconductor, with the external magnetic field applied parallel to this current ${ }^{17} 19$, allowing measurement of the longitudinal resistivity. It has been known for some time that the spontaneous production of finite vortex loops at non-zero temperatures can be a source of non-linear resistivity in the Meissner-phase of any superconductored. This idea has been extended to the problem of the resistivity along the field direction in the mixed state of a type-II superconductor 15 . If one projects the vortex lines onto the $x-y$ plane, then entanglements of the vortices may be identified as 'planar loops' perpendicular to the field direction. For any current, $J_{z}$, flowing in this direction, these planar loops will have an interaction with the current analogous to the vortex loops in the Meissner-phase. The braid defects that we have calculated are precisely the sort of defect that project to a planar loop.

It is important to distinguish between two different limiting cases. The case we consider 
is where the vortex-lattice contains 'weak entanglement' only, i.e. entanglements produced by the presence of the current itself. This should be distinguished from 'strong entanglement' where planar loop type entanglements will exist at equilibrium, on all length scales, which gives rise to a linear longitudinal resistivity 15 . However, we have shown that in a large region of the phase diagram, there are very few entanglements present in the size of samples used in experiments, so that the systems are always in the weak entanglement limit. The fact that the resistivity has been observed to become non-linear below a line much higher than the irreversibility line in $\mathrm{YBCO}^{19}$ supports our claims in Section $\mathrm{VA}$ concerning the absence of entanglements.

Following the arguments of Feigelman et. al. costs of the relevant defects, we find that in the presence of a current density $J_{z}$, a large braid has a total free energy cost of:

$$
\Delta F\left(A, J_{z}\right)=\epsilon_{A} A^{\frac{3}{4}}-\phi_{0} J_{z} A,
$$

with $\epsilon_{A} \simeq 2.1 l^{-3 / 2} k_{B} T\left|\alpha_{T}\right|^{3 / 2}=2.3\left(B / \phi_{0}\right)^{3 / 4} k_{B} T\left|\alpha_{T}\right|^{3 / 2}$. Therefore there will be a critical area, $A_{c}\left(J_{z}\right)$, above which the braid will be driven to larger growth by the magnetic interaction with the current:

$$
A_{c} \simeq 8.75 \frac{\left(k_{B} T\right)^{4} \alpha_{T}{ }^{6} B^{3}}{\phi_{0}{ }^{7} J_{z}{ }^{4}} .
$$

This will cause dissipation, which will be proportional to the probability of nucleating large enough braids by thermal fluctuations. The nucleation of large enough braids requires jumping a free energy barrier, which will lead to a longitudinal resistivity of the form:

$$
\rho_{z z}=\frac{E_{z}}{J_{z}} \propto e^{-\left(\frac{J_{T}}{J_{z}}\right)^{3}}
$$

with $J_{T} \simeq 1.43\left(B / \phi_{0}^{2}\right) k_{B} T \alpha_{T}^{2}$. This form is quite different to that given previously where it was assumed that the free energy cost of planar loops had a linear dependence on their radius.

Unfortunately, the applicability of the above results to any experiments that measure this longitudinal resistivity is rather doubtful. This is because at regions of the $H$ - $T$ phase diagram of interest, and for all reasonable current densities, the critical radius of a braid that needs to be created is far larger than the magnetic penetration depth $\lambda_{a b}$, (i.e. the length scale in the $a b$ plane over which the magnetic field may vary significantly). Therefore, the important braid defects will be outside the scale where the LLL approximation may hold.

\section{Crossing Defects as Energy Barriers to Disentanglement}

The energy barrier $U_{\times}$to the crossing of two vortices, or to a process of cutting and reconnection, is the energy cost of the configuration with the highest free energy that must exist during these processes. This configuration is assumed to be where the two lines meet each other at a point, as in the crossing defects that we have calculated. Therefore, our result for $\Delta F_{2 \times}$ for the free energy cost of a two-line crossing defect maybe used as an estimate of 
$U_{\times}$. The value of $U_{\times}$has been shown to be an important parameter of the entangled vortex state 3 . The relaxation time of the entangled vortices grows exponentially with $U_{\times} / k_{B} T$. A different form for the longitudinal resistivity to (5.3) that applies when there is strong entanglement 1 (i.e. braids exist on all length scales) is proportional to $\exp \left(-U_{\times} / k_{B} T\right.$ ) (as crossing defects will be the basic energy barrier to the growth of braids). It is doubtful where this applies though, as we have shown above that the vortex state is not entangled over a large region of the $H$ - $T$ phase diagram.

Estimates of $U_{\times}$have also been used 5 to explain the transition from local to non-local resistivity seen in the DC flux transformer experiments on YBCO 18 , 19 . In this interpretation, the average length over which crossing defects take place is associated with a correlation length of vortex 'identity' 5 . When this length scale becomes smaller than the system size, the vortices will be uncorrelated between the top and bottom of the sample, and non-local effects will be of less importance in the resistivity. This argument does not depend on the entanglement of the vortices.

\section{SUMMARY}

To summarize, we have calculated the free energy costs of an assortment of topological defects of the Abrikosov vortex-lattice that is the mean-field solution for the Ginzburg-Landau free energy functional of a perfect bulk Type-II superconductor. All of our calculations are made within the lowest Landau level, which is a good approximation at high fields just below the $H_{c 2}$ line. Although we have made a large approximation in not allowing the surrounding lattice to move in response to these defects, we believe that our answers are still very close to the full solutions with relaxation included. This is a consequence of the elastic line nature of the lattice, whereby the vortices' resistance to tilting holds them near to their ground state positions.

The free energy costs calculated are for crossing defects of 2, 3, 6, and 12 lines; braid defects of 6 and 12 lines (see Table [V]); infinite screw defects and a form for very large braids. Within the LLL, one cannot truly view the vortex system in terms of discrete lines with simple two-body interactions between them (as is the case in the opposite extremes of the London limit). This non-trivial nature of vortex-vortex interactions has led to a few unexpected results of our calculations. Firstly there were no braid solutions for two- or threeline defects - if any braid configuration of two/three lines is imposed it may continuously lower its energy by the lines meeting each other at a point (and thus forming a crossing defect)! Secondly, we have found a lower free energy cost for three lines crossing than for a two-line crossing. One assumes that this is a consequence of the lattice symmetry, together with the effect stated above that the surrounding lines in the lattice do not relax much in response to a localized defect. Thirdly, it costs a lower free energy for a six-line crossing defect than a six-line braid, even though the potential terms are higher for the crossing defect (the tilt terms decrease when the six lines move nearer each other). This odd result was not carried through to the twelve-line defects, and we believe that all larger braids cost less energy than the associated crossings. Finally, we found that for large screw defects, the free energy cost diverges with the system size perpendicular to the defect. This effect (a long range dependence in the tilt energy) leads to a result for the free energy cost of large braids which depends on the area enclosed by the braid in a new way. 
We think that our results will be useful in interpreting experiments on Type-II superconductors where the dynamics of the vortices play an important role, at least in the regimes of high field, and where any layered structure of the superconductors may be ignored (i.e. as long as the coherence length $\xi_{c}$ is greater than the inter-layer thickness). Some particular areas of application were outlined in Section $\nabla$, but there are still some remaining questions yet to be cleared up. For instance, what if any are the consequences of a lower energy cost of crossing for three-lines than two-lines? We have not yet been able to use the energy costs calculated to make quantitative predictions on transport properties such as the resistivity. However, we have shown that the equilibrium vortex state will not be entangled over a significant region above and below the irreversibility line of a high- $T_{c}$ superconductor. This prediction is open to experimental test, possibly with a magnetic probe like neutron scattering.

\section{ACKNOWLEDGEMENTS}

One of us (MJWD) would like to thank the EPSRC for funding this research.

\section{APPENDIX A: PRODUCT FORM OF THE JACOBI-THETA FUNCTION}

In this appendix, it is shown explicitly that $f(z)=\vartheta_{3}(\pi z / l, \pi \tau / l)$ has the form of (2.7). This then justifies our procedure in forming a new function with displaced zeros, but which is still in the LLL subspace, as is done in (2.11). Starting from the standard product representation of a Jacobi-theta function 11 , and for clarity letting $l=1$, we have

$$
\vartheta_{3}(\pi z, \pi \tau)=\Theta \prod_{n=0}^{\infty}\left(1-e^{2 \pi i\left(z-z_{n}\right)}\right)\left(1-e^{-2 \pi i\left(z+z_{n}\right)}\right)
$$

where $\Theta=\prod_{n=0}^{\infty}\left(1-e^{2 \pi i n \tau}\right)=$ const, and $z_{n}=-\left(n+\frac{1}{2}\right) \tau-\frac{1}{2}$. (For the triangular lattice, $\tau=1 / 2+i \sqrt{3} / 2$ and $\Theta \simeq 1.0043$.) This is the form that was used to find $\psi_{0}$ when numerically calculating the coefficients of Tables 표피. Now, the two products in (A1) can be rewritten as $\left(1-\exp \left[ \pm 2 \pi i\left(z \mp z_{n}\right)\right]\right)=\mp 2 i \exp \left[ \pm \pi i\left(z \mp z_{n}\right)\right] \sin \pi\left(z \mp z_{n}\right)$. Substituting this expression into (A1) gives

$$
\vartheta_{3}(\pi z, \pi \tau)=\Theta \prod_{n=0}^{\infty} 4 e^{-2 i \pi z_{n}} \sin \pi\left(z-z_{n}\right) \sin \pi\left(z+z_{n}\right) .
$$

The prefactor, $\prod_{n=0}^{\infty} 4 \exp \left(-2 i \pi z_{n}\right)$, can be seen to have the correct form to ensure convergence of the product, because as $n \rightarrow \infty$, for finite $z$, we get: $\sin \pi\left(z-z_{n}\right) \sin \pi\left(z+z_{n}\right) \simeq$ $(1 / 4) \exp \left(2 i \pi z_{n}\right)$. Therefore for large $n$, the terms inside the product tend to unity (as is necessary for an infinite product). Now we can use the well known product representation of $\sin x=x \prod_{n=1}^{\infty}\left(1-\left(x^{2} / n^{2} \pi^{2}\right)\right)$. Putting the sine terms in ( $\left.\mathrm{A2}\right)$ in to this form finally reveals the product form we are looking for:

$$
\vartheta_{3}(\pi z, \pi \tau)=\pi^{2} \Theta \prod_{n=0}^{\infty} 4 e^{-2 i \pi z_{n}}\left(z+z_{n}\right)\left(z-z_{n}\right) \prod_{i, j=1}^{\infty}\left(1-\frac{\left(z+z_{n}\right)^{2}}{i^{2}}\right)\left(1-\frac{\left(z-z_{n}\right)^{2}}{j^{2}}\right) .
$$


Each of the terms in the second product may be expanded as $1-\left(z \pm z_{n}\right)^{2} / j^{2}=$ $\left(1 / j^{2}\right)\left(j+\left(z \pm z_{n}\right)\right)\left(j-\left(z \pm z_{n}\right)\right)$. This is proportional to the product of two terms of the form $\left(z-z_{j}\right)$, where the roots, $z_{j}$, are given by

$$
\begin{aligned}
z_{j} & =\mp z_{n} \pm j \\
& = \pm\left(\frac{\tau+1}{2}+n \tau\right) \pm j .
\end{aligned}
$$

Therefore, we have shown that $f(z)=\vartheta_{3}(\pi z / l, \pi \tau / l)$ has the general product form of (2.7) and the zeros are positioned periodically in the complex plane, with periods $\tau$ and 1 . 


\section{REFERENCES}

${ }^{1}$ G Eilenberger, Phys. Rev., 164(2), 628 (1967).

${ }^{2}$ A L Fetter and P C Hohenberg, in Superconductivity, edit. R D Parks (Dekker, New York, 1969), Vol 2.

${ }^{3}$ M E Cates, Phys. Rev. B,45 (21), 12415 (1992).

${ }^{4}$ M C Marchetti and D Nelson, Phys. Rev. B, 42 (16), 9938 (1990).

${ }^{5}$ N K Wilkin and M A Moore, Phys. Rev. B., 50(14), 10294, (1994)

${ }^{6} \mathrm{C}$ Carraro and D S Fisher, Preprint, (1994)

${ }^{7}$ N K Wilkin and M A Moore, Phys. Rev. B., 48, 3464 (1993)

${ }^{8}$ D R Nelson, Journ. of Stat. Phys., 57(3-4), 511 (1989)

${ }^{9}$ G J Ruggeri and D J Thouless, J. Phys. F: Metal Phys., 6 (11), 2063 (1976).

${ }^{10}$ E H Brandt, Phys. Stat. Sol., 36, 381 (1969).

${ }^{11}$ G Blatter, M V Feigelman, V B Geshkenbein, A I Larkin and V M Vinokur, To be Published in Rev. Mod. Phys.

${ }^{12}$ R Labusch, Phys. Lett. 22, 9 (1966)

${ }^{13}$ M C Marchetti and D Nelson, Phys. Rev. B, 41 (4), 1910 (1990).

${ }^{14}$ D S Fisher, M P A Fisher and D A Huse, Phys. Rev. B., 43, 130 (1991).

${ }^{15}$ M V Feigelman, V B Geshkenbein, L B Ioffe and A I Larkin, Phys. Rev. B., 48(22), 16641 (1993)

16 Z Yao, S Yoon, H Dai, S Fan and C M Lieber, Nature 371, 777 (1994)

${ }^{17}$ R Busch, G Ries, H Werthner, G Kreiselmeyer and G Saemann-Ischenko, Phys. Rev. Lett., 69(3), 522 (1992).

${ }^{18}$ H Safar, P L Gammel, D A Huse, S N Majumdar, L F Schneemeyer and D J Bishop, Phys. Rev. Lett., 72(8), 1272 (1994).

${ }^{19}$ F de la Cruz, D Lopez and G Nieva, Phil. Mag. B., 70(3), 773 (1994)

${ }^{20} \mathrm{~J}$ S Langer and M E Fisher, Phys. Rev. Lett., 19, 560 (1967)

21 Table of Integrals, Series, and Products, I S Gradshteyn and I M Ryzhik (Academic Press, 1965). 
TABLE I. Coefficients for two lines allowed to move, $c_{i j}^{(2)}$

\begin{tabular}{lccccc}
\hline \hline$i$ & 0 & 1 & 2 & 3 & 4 \\
\hline$c_{i 0}^{(2)}$ & 0.7905 & -5.201 & 10.0395 & -32.7365 & 100.8234 \\
$c_{i 1}^{(2)}$ & 5.201 & 5.1655 & -32.7365 & 403.2937 & \\
$c_{i 2}^{(2)}$ & 10.0395 & 32.7365 & 604.9406 & & \\
$c_{i 3}^{(2)}$ & 32.7365 & 403.2937 & & & \\
$c_{i 4}^{(2)}$ & 100.8234 & & & & \\
\hline \hline
\end{tabular}

TABLE II. Coefficients for two lines crossing with two lines relaxing, $c_{i j}^{(4)}, u_{i}, v_{i}, w_{i j}$

\begin{tabular}{cccccc}
\hline \hline$i$ & 0 & 1 & 2 & 3 & 4 \\
\hline$c_{i 0}^{(4)}$ & 2.0304 & 6.2416 & 8.6774 & 5.9207 & 3.3321 \\
$c_{i 1}^{(4)}$ & -6.2416 & -19.9304 & -27.4202 & -33.9090 & -7.5801 \\
$c_{i 2}^{(4)}$ & 8.6774 & 27.4202 & 40.8076 & 39.0456 & 48.8558 \\
$c_{i 3}^{(4)}$ & -5.9207 & -33.9090 & -39.0456 & -103.4540 & -26.7255 \\
$c_{i 4}^{(4)}$ & 3.3321 & 7.5801 & 48.8558 & 26.7255 & 274.8685 \\
\hline$u_{i}$ & 28.7674 & -13.9138 & 28.7674 & & \\
$v_{i}$ & 28.7674 & 13.9138 & 134.871 & & \\
$w_{i 0}$ & 57.5348 & -13.9138 & & & \\
$w_{i 1}$ & 13.9138 & 269.742 & & & \\
\hline \hline
\end{tabular}

TABLE III. Coefficients for three and six lines allowed to move, $c_{i j k}^{(3)}, c_{i j k}^{(6)}$

\begin{tabular}{cccccccc}
\hline \hline$i j k$ & 000 & 200 & 400 & 110 & 220 & 202 & 310 \\
\hline$c_{i j k}^{(3)}$ & 0.5443 & -23.7064 & 293.7881 & -2.0347 & 11.6525 & 11.5790 & -15.3239 \\
$c_{i j k}^{(6)}$ & 1.40174 & -2.8908 & 1.32786 & -0.18099 & 0.37540 & 0.38420 & -0.03321 \\
\hline \hline
\end{tabular}

TABLE IV. Summary of the Free Energy costs of Crossing and Braid Defects

\begin{tabular}{ccccc}
\hline \hline$i$ & 2 & 3 & 6 & 12 \\
\hline$\Delta F_{i \times} /\left(k_{B} T\left|\alpha_{T}\right|^{\frac{3}{2}}\right)$ & 0.58 & 0.51 & 1.38 & 8.6 \\
$\Delta F_{i b r} /\left(k_{B} T\left|\alpha_{T}\right|^{\frac{3}{2}}\right)$ & & & 2.19 & 6.5 \\
\hline \hline
\end{tabular}

